\title{
Measurement of charged particle spectra in deep-inelastic ep scattering at HERA
}

The H1 Collaboration

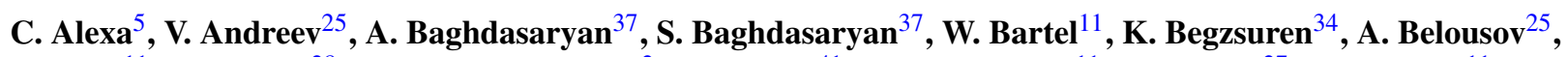
P. Belov ${ }^{11}$, V. Boudry ${ }^{28}$, I. Bozovic-Jelisavcic ${ }^{2}$, G. Brandt ${ }^{41}$, M. Brinkmann ${ }^{11}$, V. Brisson ${ }^{27}$, D. Britzger $^{11}$, A. Buniatyan ${ }^{14}$, A. Bylinkin ${ }^{24, g}$, L. Bystritskaya ${ }^{24}$, A.J. Campbell ${ }^{11}$, K.B. Cantun Avila ${ }^{22}$, F. Ceccopieri ${ }^{4}$, K. Cerny ${ }^{31}$, V. Chekelian ${ }^{26}$, J.G. Contreras ${ }^{22}$, J. Cvach $^{30}$, J.B. Dainton ${ }^{18}$, K. Daum ${ }^{36, a, b}$, E.A. De Wolf ${ }^{4}$, C. Diaconu ${ }^{21}$, M. Dobre ${ }^{5}$, V. Dodonov ${ }^{13}$, A. Dossanov ${ }^{12,26}$, G. Eckerlin ${ }^{11}$, S. Egli ${ }^{35}$, E. Elsen ${ }^{11}$, L. Favart ${ }^{4}$, A. Fedotov Fe $^{24}$ R. Felst $^{11}$, J. Feltesse $^{10}$, J. Ferencei ${ }^{16}$, D.-J. Fischer ${ }^{11}$, M. Fleischer ${ }^{11}$, A. Fomenko ${ }^{25}$, E. Gabathuler ${ }^{18}$, J. Gayler $^{11}$, S. Ghazaryan ${ }^{11}$, A. Glazov ${ }^{11}$, L. Goerlich ${ }^{7}$, N. Gogitidze ${ }^{25}$, M. Gouzevitch ${ }^{11, c}$, C. Grab ${ }^{39}$, A. Grebenyuk $^{11}$, T. Greenshaw ${ }^{18}$, G. Grindhammer ${ }^{26}$, S. Habib ${ }^{11}$, D. Haidt ${ }^{11}$, R.C.W. Henderson ${ }^{17}$, E. Hennekemper ${ }^{15}$, M. Herbst $^{15}$, G. Herrera ${ }^{23}$, M. Hildebrandt ${ }^{35}$, K.H. Hiller ${ }^{38}$, J. Hladkì ${ }^{30}$, D. Hoffmann ${ }^{21}$, R. Horisberger ${ }^{35}$, T. Hreus $^{4}$, F. Huber ${ }^{14}$, M. Jacquet ${ }^{27}$, X. Janssen ${ }^{4}$, L. Jönsson ${ }^{20}$, H. Jung ${ }^{11,4}$, M. Kapichine ${ }^{9}$, C. Kiesling ${ }^{26}$, M. Klein ${ }^{18}$, C. Kleinwort ${ }^{11}$, R. Kogler ${ }^{12}$, P. Kostka ${ }^{38}$, M. Krämer ${ }^{11}$, J. Kretzschmar ${ }^{18}$, K. Krüger ${ }^{11}$, M.P.J. Landon ${ }^{19}$,

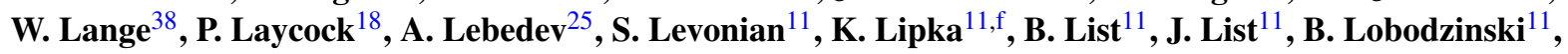
R. Lopez-Fernandez ${ }^{23}$, V. Lubimov ${ }^{24, \dagger}$, E. Malinovski ${ }^{25}$, H.-U. Martyn ${ }^{1}$, S.J. Maxfield ${ }^{18}$, A. Mehta ${ }^{18}$, A.B. Meyer ${ }^{11}$, H. Meyer $^{36}$, J. Meyer $^{11}$, S. Mikocki ${ }^{7}$, I. Milcewicz-Mika ${ }^{7}$, A. Morozov ${ }^{9}$, J.V. Morris ${ }^{6}$, K. Müller ${ }^{40}$, Th. Naumann $^{38}$, P.R. Newman ${ }^{3}$, C. Niebuhr ${ }^{11}$, D. Nikitin ${ }^{9}$, G. Nowak ${ }^{7}$, K. Nowak ${ }^{12, f}$, J.E. Olsson ${ }^{11}$, D. Ozerov ${ }^{11}$, P. Pahl ${ }^{11}$, V. Palichik ${ }^{9}$, M. Pandurovic ${ }^{2}$, C. Pascaud ${ }^{27}$, G.D. Patel ${ }^{18}$, E. Perez ${ }^{10, d}$, A. Petrukhin ${ }^{11}$, I. Picuric ${ }^{29}$, H. Pirumov $^{14}$,

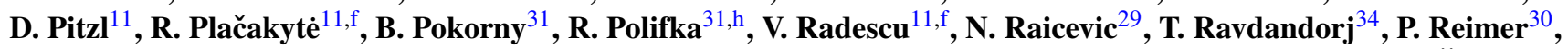
E. Rizvi ${ }^{19}$, P. Robmann ${ }^{40}$, R. Roosen ${ }^{4}$, A. Rostovtsev ${ }^{24}$, M. Rotaru ${ }^{5}$, J.E. Ruiz Tabasco ${ }^{22}$, S. Rusakov $^{25}$, D. Š́lek $^{31}$, D.P.C. Sankey ${ }^{6}$, M. Sauter ${ }^{14}$, E. Sauvan ${ }^{21, i}$, S. Schmitt ${ }^{11}$, L. Schoeffel ${ }^{10}$, A. Schöning ${ }^{14}$, H.-C. Schultz-Coulon ${ }^{15}$, F. Sefkow ${ }^{11}$, S. Shushkevich ${ }^{11}$, Y. Soloviev ${ }^{11,25}$, P. Sopicki ${ }^{7}$, D. South ${ }^{11}$, V. Spaskov ${ }^{9}$, A. Specka ${ }^{28}$, Z. Staykova ${ }^{4}$, M. Steder ${ }^{11}$, B. Stella ${ }^{32}$, G. Stoicea ${ }^{5}$, U. Straumann ${ }^{40}$, T. Sykora ${ }^{4,31}$, P.D. Thompson ${ }^{3}$, D. Traynor ${ }^{19}$, P. Truöl ${ }^{40}$, I. Tsakov ${ }^{33}$, B. Tseepeldorj ${ }^{24, e}$, J. Turnau $^{7}$, A. Valkárová ${ }^{31}$, C. Vallée $^{21}$, P. Van Mechelen ${ }^{4}$, Y. Vazdik ${ }^{25}$, D. Wegener $^{8}$, E. Wünsch ${ }^{11}$, J. Žáček ${ }^{31}$, J. Zálešák ${ }^{30}$, Z. Zhang ${ }^{27}$, R. Žlebčík ${ }^{31}$, H. Zohrabyan ${ }^{37}$, F. Zomer ${ }^{27}$

${ }^{1}$ I. Physikalisches Institut der RWTH, Aachen, Germany

${ }^{2}$ Vinca Institute of Nuclear Sciences, University of Belgrade, 1100 Belgrade, Serbia

${ }^{3}$ School of Physics and Astronomy, University of Birmingham, Birmingham, UK ${ }^{\mathrm{k}}$

${ }^{4}$ Inter-University Institute for High Energies ULB-VUB, Brussels and Universiteit Antwerpen, Antwerpen, Belgium ${ }^{1}$

${ }^{5}$ National Institute for Physics and Nuclear Engineering (NIPNE), Bucharest, Romania ${ }^{t}$

${ }^{6}$ STFC, Rutherford Appleton Laboratory, Didcot, Oxfordshire, UK ${ }^{\mathrm{k}}$

${ }^{7}$ Institute for Nuclear Physics, Cracow, Poland ${ }^{\mathrm{m}}$

${ }^{8}$ Institut für Physik, TU Dortmund, Dortmund, Germany ${ }^{\mathrm{j}}$

${ }_{9}^{9}$ Joint Institute for Nuclear Research, Dubna, Russia

${ }^{10} \mathrm{CEA}, \mathrm{DSM} / \mathrm{Irfu}, \mathrm{CE}-$ Saclay, Gif-sur-Yvette, France

${ }^{11}$ DESY, Hamburg, Germany

${ }^{12}$ Institut für Experimentalphysik, Universität Hamburg, Hamburg, Germany ${ }^{j}$

${ }^{13}$ Max-Planck-Institut für Kernphysik, Heidelberg, Germany

${ }^{14}$ Physikalisches Institut, Universität Heidelberg, Heidelberg, Germany ${ }^{j}$

${ }^{15}$ Kirchhoff-Institut für Physik, Universität Heidelberg, Heidelberg, Germany ${ }^{j}$

${ }^{16}$ Institute of Experimental Physics, Slovak Academy of Sciences, Košice, Slovak Republic ${ }^{\text {n}}$

${ }^{17}$ Department of Physics, University of Lancaster, Lancaster, UK ${ }^{\mathrm{k}}$

${ }^{18}$ Department of Physics, University of Liverpool, Liverpool, $\mathrm{UK}^{\mathrm{k}}$

${ }^{19}$ School of Physics and Astronomy, Queen Mary, University of London, London, UK ${ }^{\mathrm{k}}$

${ }^{20}$ Physics Department, University of Lund, Lund, Sweden ${ }^{\circ}$

${ }^{21}$ CPPM, Aix-Marseille Univ, CNRS/IN2P3, 13288 Marseille, France

${ }_{22}^{22}$ Departamento de Fisica Aplicada, CINVESTAV, Mérida, Yucatán, México ${ }^{r}$

${ }^{23}$ Departamento de Fisica, CINVESTAV IPN, México City, México ${ }^{\mathrm{r}}$

${ }^{24}$ Institute for Theoretical and Experimental Physics, Moscow, Russias 
${ }^{25}$ Lebedev Physical Institute, Moscow, Russia

${ }^{26}$ Max-Planck-Institut für Physik, München, Germany

${ }^{27}$ LAL, Université Paris-Sud, CNRS/IN2P3, Orsay, France

${ }^{28}$ LLR, Ecole Polytechnique, CNRS/IN2P3, Palaiseau, France

${ }^{29}$ Faculty of Science, University of Montenegro, Podgorica, Montenegro ${ }^{\mathrm{u}}$

${ }^{30}$ Institute of Physics, Academy of Sciences of the Czech Republic, Praha, Czech Republic ${ }^{p}$

${ }^{31}$ Faculty of Mathematics and Physics, Charles University, Praha, Czech Republic ${ }^{p}$

${ }^{32}$ Dipartimento di Fisica Università di Roma Tre and INFN Roma 3, Roma, Italy

${ }^{33}$ Institute for Nuclear Research and Nuclear Energy, Sofia, Bulgaria

${ }^{34}$ Institute of Physics and Technology of the Mongolian Academy of Sciences, Ulaanbaatar, Mongolia

${ }^{35}$ Paul Scherrer Institut, Villigen, Switzerland

${ }^{36}$ Fachbereich C, Universität Wuppertal, Wuppertal, Germany

${ }^{37}$ Yerevan Physics Institute, Yerevan, Armenia

${ }^{38}$ DESY, Zeuthen, Germany

${ }^{39}$ Institut für Teilchenphysik, ETH, Zürich, Switzerland ${ }^{q}$

${ }^{40}$ Physik-Institut der Universität Zürich, Zürich, Switzerland ${ }^{q}$

${ }^{41}$ Department of Physics, Oxford University, Oxford, UK ${ }^{\mathrm{k}}$

Received: 6 February 2013 / Revised: 28 March 2013 / Published online: 20 April 2013

(C) The Author(s) 2013. This article is published with open access at Springerlink.com

\begin{abstract}
Charged particle production in deep-inelastic ep scattering is measured with the $\mathrm{H} 1$ detector at HERA. The kinematic range of the analysis covers low photon virtualities, $5<Q^{2}<100 \mathrm{GeV}^{2}$, and small values of Bjorken- $x$, $10^{-4}<x<10^{-2}$. The analysis is performed in the hadronic centre-of-mass system. The charged particle densities are measured as a function of pseudorapidity $\left(\eta^{*}\right)$ and transverse momentum $\left(p_{T}^{*}\right)$ in the range $0<\eta^{*}<5$ and $0<$ $p_{T}^{*}<10 \mathrm{GeV}$ in bins of $x$ and $Q^{2}$. The data are compared to predictions from different Monte Carlo generators implementing various options for hadronisation and parton evolutions.
\end{abstract}

a e-mail: daum@mail.desy.de

${ }^{\mathrm{b}}$ Also at Rechenzentrum, Universität Wuppertal, Wuppertal, Germany.

${ }^{\mathrm{c}}$ Also at IPNL, Université Claude Bernard Lyon 1, CNRS/IN2P3, Villeurbanne, France.

${ }^{\mathrm{d}}$ Also at CERN, Geneva, Switzerland.

${ }^{\mathrm{e}}$ Also at Ulaanbaatar University, Ulaanbaatar, Mongolia.

${ }^{\text {f }}$ Supported by the Initiative and Networking Fund of the Helmholtz Association (HGF) under the contract VH-NG-401 and S0-072.

${ }^{\mathrm{g}}$ Also at Moscow Institute of Physics and Technology, Moscow, Russia.

${ }^{\mathrm{h}}$ Also at Department of Physics, University of Toronto, Toronto, Ontario, Canada M5S 1A7.

${ }^{\mathrm{i}}$ Also at LAPP, Université de Savoie, CNRS/IN2P3, Annecy-le-Vieux, France.

${ }^{\mathrm{j}}$ Supported by the Bundesministerium für Bildung und Forschung, FRG, under contract numbers 05H09GUF, 05H09VHC, 05H09VHF, 05H16PEA.

${ }^{\mathrm{k}}$ Supported by the UK Science and Technology Facilities Council, and formerly by the UK Particle Physics and Astronomy Research Council.

${ }^{1}$ Supported by FNRS-FWO-Vlaanderen, IISN-IIKW and IWT and by Interuniversity Attraction Poles Programme, Belgian Science Policy.

\section{Introduction}

Deep-inelastic scattering (DIS) processes at the $e p$ collider HERA can access small values of Bjorken- $x$ at low four momentum transfers squared $Q^{2}$ of a few $\mathrm{GeV}^{2}$. In the region of low $x$, characterised by high densities of gluons and sea quarks in the proton, the parton interaction with the virtual photon may originate from a cascade of partons emitted prior to the interaction as illustrated in Fig. 1. In perturbative Quantum Chromodynamics (QCD) such multi-parton emissions are described only within certain approximations valid in restricted phase space regions. At sufficiently large $Q^{2}$ and not too small $x$ the Dokshitzer-Gribov-LipatovAltarelli-Parisi (DGLAP) [1-5] evolution equation is expected to be a good approximation. The DGLAP equation corresponds to a strong ordering of the transverse momenta of the propagator partons, $k_{T, i}$, with respect to the proton direction, which implies strong ordering of the transverse momenta of the emitted partons, $p_{T, i} \ll p_{T, i+1}$, in the parton

${ }^{\mathrm{m}}$ Partially Supported by Polish Ministry of Science and Higher Education, grant DPN/N168/DESY/2009.

${ }^{\mathrm{n}}$ Supported by VEGA SR grant no. 2/7062/27.

${ }^{\circ}$ Supported by the Swedish Natural Science Research Council.

pSupported by the Ministry of Education of the Czech Republic under the projects LC527, INGO-LA09042 and MSM0021620859.

${ }^{\mathrm{q}}$ Supported by the Swiss National Science Foundation.

${ }^{\mathrm{r}}$ Supported by CONACYT, México, grant 48778-F.

${ }^{\mathrm{s}}$ Russian Foundation for Basic Research (RFBR), grant no 1329.2008.2 and Rosatom.

${ }^{\text {t}}$ Supported by the Romanian National Authority for Scientific Research under the contract PN 09370101.

upartially Supported by Ministry of Science of Montenegro, no. 051/3-3352.

$\dagger$ Deceased. 


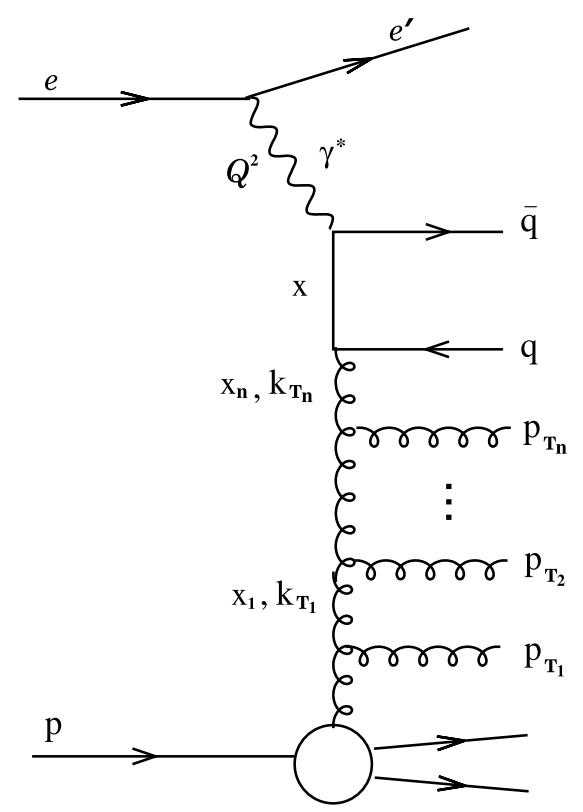

Fig. 1 Generic diagram for deep-inelastic ep scattering at small $x$. The transverse momenta of the emitted gluons are labeled as $p_{T, i}$, while the proton longitudinal momentum fractions and the transverse momenta carried by the propagating gluons are denoted by $x_{i}$ and $k_{T, i}$, respectively

cascade from the proton towards the virtual photon. At small $x$ the DGLAP approximation is expected to become inadequate and the Balitsky-Fadin-Kuraev-Lipatov (BFKL) [6-8] scheme may be more appropriate, which has no ordering in $k_{T}$ of the partons along the ladder. The CiafaloniCatani-Fiorani-Marchesini (CCFM) evolution [9-12] aims to unify the DGLAP and BFKL approaches. It introduces angular ordering of gluon emissions to implement coherence effects. At small $x$ the CCFM evolution equation is almost equivalent to the BFKL approach, while it reproduces the DGLAP equations for sufficiently large $x$ and $Q^{2}$.

Measurements of the proton structure function $F_{2}\left(x, Q^{2}\right)$ $[13,14]$ are well described by the Next-to-Leading-Order (NLO) or Next-to-Next-to-Leading Order (NNLO) DGLAP evolution [15-25], suggesting that this observable may be too inclusive to exhibit signals for BFKL dynamics. Deviations from the $k_{T}$ ordering at HERA are observed in jet production [26, 27], transverse energy flow [28, 29], forward jet production [30-32] and measurements of forward $\pi^{0}$ production [33]. Studies of the transverse momentum spectrum of charged particles have been proposed in [34] as a more direct probe of the underlying parton dynamics. In that paper it has been shown with the help of QCD models that the high- $p_{T}$ tail is sensitive to parton radiation, while the contribution from hadronisation is small. Previous measurements of the transverse momentum and pseudorapidity dependence of particle densities performed by the H1 collaboration [35] were limited in statistical precision.
This paper presents a study of charged particle production in $e p$ collisions for $5<Q^{2}<100 \mathrm{GeV}^{2}$. The analysis is performed in the hadronic centre-of-mass frame, i.e. in the virtual photon-proton rest frame. The charged particle densities as a function of pseudorapidity and transverse momentum are measured differentially in $x$ and $Q^{2}$.

The data set used for the analysis was collected with the H1 detector in 2006 when positrons and protons collided with energies of $27.6 \mathrm{GeV}$ and $920 \mathrm{GeV}$, respectively, corresponding to a centre-of-mass energy of $\sqrt{s}=319 \mathrm{GeV}$. The integrated luminosity of the data set is $88.6 \mathrm{pb}^{-1}$, which is about seventy times larger than what was available for the previous $\mathrm{H} 1$ measurement [35]. This allows for a more detailed study of the dynamical features of parton evolution in the proton at small $x$.

\section{QCD models}

Parton cascade and hadronisation processes leading to charged particle production in $e p$ collisions are modeled using different Monte Carlo (MC) programs. Brief descriptions of the $\mathrm{MC}$ event generators considered in this analysis are given below.

- The RAPGAP [36] MC generator matches first order QCD matrix elements to DGLAP based leading logarithm approximations for parton showers with strongly ordered transverse momenta of subsequently emitted partons. The factorisation and renormalisation scales are set to $\mu_{f}=$ $\mu_{r}=\sqrt{Q^{2}+{\hat{p_{T}}}^{2}}$, where $\hat{p}_{T}$ is the transverse momentum of the outgoing hard parton from the matrix element in the centre-of-mass frame of the hard subsystem.

- The DJAngoh [37] MC generator uses the Colour Dipole Model (CDM) as implemented in ARIADNE [38], which models first order QCD processes and creates dipoles between coloured partons. Gluon emission is treated as radiation from these dipoles, and new dipoles are formed from the emitted gluons from which further radiation is possible. The radiation pattern of the dipoles includes interference effects, thus modelling gluon coherence. The transverse momenta of the emitted partons are not ordered, producing a configuration similar to the BFKL treatment of parton evolution [39, 40].

- The CASCADE [41, 42] MC generator uses off-shell leading order QCD matrix elements, supplemented with parton emissions based on the CCFM evolution equation. The equation requires an unintegrated gluon density (see $[41,42]$ ), which takes the transverse momenta of the propagators into account. In contrast to the DGLAP evolution equation, the CCFM equation only contains gluon splitting $g \rightarrow g g$. 
- The Herwig++ [43] MC program with the POWHEG (POsitive Weight Hardest Emission) option [44], combines the full matrix element including virtual corrections at $\mathcal{O}\left(\alpha_{s}\right)$ with a DGLAP-like parton shower simulation. The Herwig++ MC program uses the Coherent Parton Branching algorithm $[45,46]$, which implements coherence via ordering in $\hat{q}$ which is defined as $\hat{q}=p_{T} /(1-z)$ and $\hat{q}=p_{T} /(z(1-z))$ for initial and final state showering, respectively [47]. Here $(1-z)$ is the light-cone momentum fraction of the emitted parton and $p_{T}$ is the transverse momentum of the splitting products.

- Photoproduction background is generated with the PHOJET $[48,49]$ program, which uses a two-component dual parton model [50] including diffractive processes and vector meson production.

The RAPGAP and DJANGOH predictions are calculated using the CTEQ6L(LO) [51] set of Parton Distribution Functions (PDF), while CASCADE uses the default A0 unintegrated gluon density set [52]. The predictions of Herwig++ were obtained with the default PDF MRST 02 NLO [53]. To simulate hadronisation the Lund string fragmentation model [54] is used, as implemented in JETSET [55] for DJANGOH and PYTHIA [56] for both RAPGAP and CASCADE. The parameters of the Lund string fragmentation model used here are tuned to describe $e^{+} e^{-}$results [57]. The tuning was performed by the ALEPH collaboration using hadronic $Z$ decay data and the PYTHIA6.1 simulation with Bose-Einstein correlations turned on. In addition, the tune obtained by the Professor tool [58] using LEP data is also tested. Herwig++ incorporates the cluster model $[59,60]$ of hadronisation, in which colour-singlet clusters of partons form after the perturbative phase and then decay into the observed hadrons.

DJANGOH and RAPGAP are also used together with the $\mathrm{H} 1$ detector simulation in order to determine the acceptance and efficiency and to estimate the systematic uncertainties associated with the measurement. The programs are interfaced to HERACLES [61] to simulate the QEDradiative effects. The generated events are passed through a detailed simulation of the $\mathrm{H} 1$ detector response based on the GEANT simulation program [62] and are processed using the same reconstruction and analysis program chain as for data. For the determination of the detector effects both the RAPGAP and DJANGOH predictions are studied. Both models describe all relevant control distributions reasonably well [63]. To improve the determination of the detector corrections the transverse momentum, pseudorapidity and multiplicity of charged particles as well as inelasticity $y$, defined as $y=Q^{2} /(s \cdot x)$, are reweighted to the data [63]. The reweighting is applied to the generator quantities.

\section{Experimental method}

\section{$3.1 \mathrm{H} 1$ detector}

A full description of the $\mathrm{H} 1$ detector can be found elsewhere [64-66] and only the components most relevant for this analysis are briefly mentioned here. The coordinate system of $\mathrm{H} 1$ is defined such that the positive $z$ axis is pointing in the direction of the proton beam (forward direction) and the nominal interaction point is located at $z=0$. The polar angle $\theta$ is then defined with respect to this axis. The pseudorapidity is defined to be $\eta=-\ln (\tan (\theta / 2))$.

Charged particles are measured within the central tracking detector (CTD) in the polar angle range $20^{\circ}<\theta<165^{\circ}$, which is also used to reconstruct the interaction vertex. The CTD comprises two large cylindrical jet chambers (CJCs), and the silicon vertex detector $[67,68]$. The CTD is operated inside a $1.16 \mathrm{~T}$ solenoidal magnetic field. The CJCs are separated by a cylindrical drift chamber which improves the $z$ coordinate reconstruction. A cylindrical multiwire proportional chamber [69], which is mainly used in the trigger, is situated inside the inner CJC. The trajectories of charged particles are measured with a transverse momentum resolution of $\sigma\left(p_{T}\right) / p_{T} \approx 0.2 \% p_{T} / \mathrm{GeV} \oplus 1.5 \%$. The forward tracking detector (FTD) [70] measures the tracks of charged particles at polar angles $6^{\circ}<\theta<25^{\circ}$. In the region of angular overlap, FTD and short CTD track segments are used to reconstruct combined tracks, extending the detector acceptance for well-reconstructed tracks. Both the CTD and the combined tracks are linked to hits in the vertex detectors: the central silicon tracker (CST) $[67,68]$, the backward silicon tracker (BST) [71] and the forward silicon tracker (FST) [72]. These detectors provide precise spatial coordinate measurements and therefore significantly improve the primary vertex spatial resolution. The CST consists of two layers of double-sided silicon strip detectors surrounding the beam pipe covering an angular range of $30^{\circ}<\theta<150^{\circ}$ for tracks passing through both layers. The BST consists of six double wheels of strip detectors measuring the transverse coordinates of charged particles. The FST design is similar to the BST and consists of five double wheels of single-sided strip detectors.

The lead-scintillating fibre calorimeter (SpaCal) [66] covering the region $153^{\circ}<\theta<177.5^{\circ}$, has electromagnetic and hadronic sections. The calorimeter is used to measure the scattered positron and the backward hadronic energy flow. The energy resolution for positrons in the electromagnetic section is $\sigma(E) / E \approx 7.1 \% / \sqrt{E / \mathrm{GeV}} \oplus 1 \%$, as determined in test beam measurements [73]. The SpaCal provides energy and time-of-flight information used for triggering purposes. A backward proportional chamber (BPC) in front of the SpaCal is used to improve the angular measurement of the scattered lepton. The liquid argon (LAr) calorimeter [74] 
covers the range $4^{\circ}<\theta<154^{\circ}$ and is used in this analysis in the reconstruction of the hadronic final state. It has an energy resolution of $\sigma(E) / E \approx 50 \% / \sqrt{E / \mathrm{GeV}} \oplus 2 \%$ for hadronic showers, as obtained from test beam measurements [75].

\subsection{Event reconstruction}

The DIS kinematics is reconstructed based on the measurement of the scattered electron and the hadronic final state (HFS) particles. In the so-called $e \Sigma$-method [76] the kinematic variables $Q^{2}, y$ and $x$ are given by:

$$
\begin{aligned}
& Q^{2}=4 E_{e} E_{e}^{\prime} \cos ^{2}\left(\frac{\theta_{e}}{2}\right), \\
& y=2 E_{e} \frac{\Sigma}{\left[\Sigma+E_{e}^{\prime}\left(1-\cos \theta_{e}\right)\right]^{2}} \quad \text { and } \quad x=\frac{Q^{2}}{s \cdot y},
\end{aligned}
$$

where $s$ is the square of the centre-of-mass energy, $E_{e}^{\prime}$ and $\theta_{e}$ the energy and polar angle of the scattered lepton, respectively, $E_{e}$ being the energy of incoming lepton and $\Sigma=\sum_{i}\left(E_{i}-p_{z, i}\right)$ where the sum runs over all hadronic final state (HFS) particles $i$. This method provides an optimum in resolution of the kinematic variables and shows only little sensitivity to QED radiative effects. The HFS particles are reconstructed using an energy flow algorithm [77-79]. This algorithm combines charged particle tracks and calorimetric energy clusters, taking into account their respective resolution and geometric overlap, into hadronic objects, while avoiding double counting of energy.

\subsection{Data selection}

DIS events were recorded using triggers based on electromagnetic energy deposits in the SpaCal calorimeter. The trigger efficiency is determined using independently triggered data. For DIS events the trigger inefficiency is negligible in the kinematic region of the analysis.

The scattered lepton, defined by the most energetic SpaCal cluster, is required to have an energy $E_{e}^{\prime}$ larger than $12 \mathrm{GeV}$. The kinematical phase space is defined by $5<Q^{2}<100 \mathrm{GeV}^{2}$ and $0.05<y<0.6$, corresponding to the geometric acceptance of the SpaCal. The upper cut on $y$ reduces background from photoproduction. In addition, $x$ is required to be in the range of $0.0001<x<0.01$.

Additional selections are made to reduce QED radiation effects and to suppress background events. The $z$ coordinate of the event vertex is required to be within 35 $\mathrm{cm}$ of the nominal interaction point. Events with high energy initial state photon radiation are rejected by requiring $35<\sum_{i}\left(E_{i}-p_{z, i}\right)<75 \mathrm{GeV}$. Here, the sum extends over all HFS particles and the scattered electron. This cut further suppresses photoproduction background events to a level of about $0.5 \%$.
The tracks used in the analysis are measured in the CTD alone (central tracks) or result from combinations of CTD and FTD information (combined tracks). Central tracks are required to have transverse momenta in the laboratory frame $p_{T}>150 \mathrm{MeV}$. The momentum of a combined track is required to be larger than $0.5 \mathrm{GeV}$ to ensure that the track has enough momentum to cross the endwall of the CJC. Both central and combined tracks are required to originate from the primary event vertex and to be in the pseudorapidity range $-2<\eta<2.5$ measured in the laboratory frame. Using only tracks assigned to the event vertex, the contributions from in-flight decays of $K_{S}^{0}, \Lambda$ and from photon conversions and from other secondary decays are reduced. Further track quality cuts [63] are applied to ensure a high purity of the track reconstruction.

\subsection{Definition of experimental observables}

The results of this analysis are presented in the hadronic centre-of-mass frame (HCM), to minimise the effect of the transverse boost from the virtual photon. The transformation to the HCM frame is reconstructed with the knowledge of the kinematic variables $Q^{2}$ and $y$ [63]. The transverse momentum and pseudorapidity of charged particles in the HCM frame are labelled as $p_{T}^{*}$ and $\eta^{*}$. Since in this frame the positive $z^{*}$ axis is defined by the direction of the virtual photon, HFS particles with $\eta^{*}>0$ belong to the current hemisphere and particles with $\eta^{*}<0$ originate from the target (proton remnant) hemisphere.

Charged particle densities as a function of transverse momentum and pseudorapidity are defined as $(1 / N)\left(\mathrm{d} n / \mathrm{d} p_{T}^{*}\right)$ and $(1 / N)\left(\mathrm{d} n / \mathrm{d} \eta^{*}\right)$, respectively. Here, $\mathrm{d} n$ is the total number of charged particles with transverse momentum (pseudorapidity) in the $\mathrm{d} p_{T}^{*}\left(\mathrm{~d} \eta^{*}\right)$ bin and $N$ denotes the number of selected DIS events. For distributions measured differentially in $x$ and $Q^{2}, \mathrm{~d} n$ and $N$ are the numbers for the respective $\left(x, Q^{2}\right)$ bin.

Hadronisation is expected to be more relevant at small transverse momenta, while the hard parton radiation is expected to contribute more significantly at high $p_{T}^{*}\left(p_{T}^{*}>\right.$ $1 \mathrm{GeV}$ ) [34]. To distinguish hadronisations effects from parton evolution signatures, the charged particle density is measured as a function of $\eta^{*}$ for $0<p_{T}^{*}<1 \mathrm{GeV}$ and for $1<p_{T}^{*}<10 \mathrm{GeV}$. The $p_{T}^{*}$ dependence of the charged particle densities is studied in two different pseudorapidity intervals, $0<\eta^{*}<1.5$ and $1.5<\eta^{*}<5$, referred to as the "central region" and "current region", respectively, as illustrated in Fig. 2. Such division approximately defines the regions where the sensitivity to the hard scatter is largest (current region), and where the parton shower models can be tested (central region). The target region, $\eta^{*}<0$, is not accessible in this analysis. 


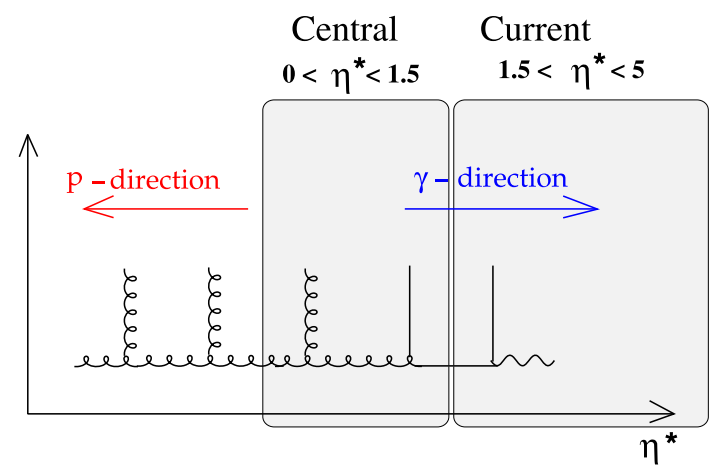

Fig. 2 The two pseudorapidity regions analysed in this paper. The region $0<\eta^{*}<1.5$ and $1.5<\eta^{*}<5$, are denoted as "central" and "current" regions, respectively

\section{Data corrections}

The data are corrected to the number of stable charged particles including charged hyperons, with proper lifetime $c \tau>10 \mathrm{~mm}$, in the phase space given in Table 1. Correction factors are calculated for each analysis bin from the ratio of the number of generated stable charged particles to the number of reconstructed tracks. The bin widths are chosen such that a purity ${ }^{1}$ of more than $75 \%$ is ensured in all bins. The correction takes into account detector effects like limited resolution and losses near the phase space boundaries, as well as a small residual contamination from weak decays of neutral particles (e.g. $K^{0}$ and $\Lambda$ ).

In addition to migrations between bins inside the measurement phase space, there are migrations from outside of the analysis phase space and there is background from photoproduction. These contributions are subtracted prior to applying the correction factors according to the procedure outlined in [32].

The DJANGOH MC was used to correct the data. The differences to the correction factors obtained from RAPGAP

Table 1 Phase space for charged particles

\begin{tabular}{ll}
\hline DIS selection & \\
Four momentum transfers squared & $5<Q^{2}<100 \mathrm{GeV}^{2}$ \\
Inelasticity & $0.05<y<0.6$ \\
Charged particles & \\
Pseudorapidity in the laboratory frame & $-2<\eta<2.5$ \\
Transverse momentum in the laboratory frame & $p_{T}>150 \mathrm{MeV}$ \\
Pseudorapidity in the HCM frame & $0<\eta^{*}<5$ \\
Transverse momentum in the HCM frame & $0<p_{T}^{*}<10 \mathrm{GeV}$ \\
\hline
\end{tabular}

\footnotetext{
${ }^{1}$ The purity is defined as the ratio of the number of charged particles generated and reconstructed in a given bin to the total number of charged particles in the phase space of the analysis which are reconstructed in this bin.
}

are taken as systematic uncertainties. The correction factors strongly depend on $\eta^{*}$ and to a lesser extent on $p_{T}^{*}$. In the $1.5<\eta^{*}<5$ region they vary between 1 and 1.8 with the largest values seen at high $p_{T}^{*}$ and large $\eta^{*}$. In the $0<\eta^{*}<1.5$ region, the correction factors rise up to 2.6 at high $p_{T}^{*}$, due to the limited detector acceptance in this region. The two MC models predict very similar correction factors for most of the phase space region, but differences up to $5.5 \%$ are observed at small $\eta^{*}$ and high $p_{T}^{*}$.

\section{Systematic uncertainties}

The following sources of systematic uncertainties are considered for all measured quantities.

- The systematic uncertainty on the SpaCal energy scale is $1 \%$ [80], which results in a systematic uncertainty of typically $0.4 \%$ for the measured single differential distributions.

- The SpaCal angular resolution of 1 mrad leads to a systematic uncertainty of about $0.1 \%$ for the measured distributions.

- The hadronic energy scale uncertainty is known to a precision of $2 \%$ [81]. The scale uncertainty enters into the uncertainty of the phase space calculations, which depend on $E-P_{z}$ of the HFS, and also affects the boost to the HCM frame. The systematic effect on the present measurements is about $0.3 \%$.

- The systematic uncertainty arising from the model dependence of the data correction is taken as the difference of the correction factors calculated using RAPGAP and DJANGOH MC. The resulting uncertainty on the measurements varies between $0.2 \%$ and $5.5 \%$.

- The systematic uncertainty associated with the track reconstruction (e.g. track reconstruction efficiency, vertex reconstruction efficiency, weak decays and nuclear interaction uncertainties) is estimated to be:

- $1 \%$ for central tracks. The track reconstruction efficiency was determined from the analysis of curling tracks and of the decay angle distribution of charged pions from the decay $K_{S}^{0} \rightarrow \pi^{+} \pi^{-}$. Data agree with the simulation within $0.5 \%$ for $p_{T}>100 \mathrm{MeV}$ [82]. Nuclear interactions of pions and kaons have been investigated by the analysis of secondary vertices of charged particles located in the material between the two CJCs and originating from interactions with the detector material. The nuclear interaction cross sections for these hadrons are found to be smaller in the simulation than in data. After correcting for these deficits, the agreement between data and $\mathrm{MC}$ in the overall track efficiency is found to be better than $1 \%$.

- $10 \%$ for combined tracks $[63,70]$. This was checked using all selected central tracks, as well as by using 
pions from $K_{S}^{0}$ decays, as a function of transverse momentum and pseudorapidity. Consistent results are obtained from both samples showing agreement of data and $\mathrm{MC}$ within $10 \%$.

The systematic uncertainty associated with the track reconstruction is applied as an independent uncorrelated uncertainty on every data point. The resulting uncertainty on the measurements varies between $1 \%$ and $5.4 \%$ and is $1.6 \%$ on average. An additional systematic uncertainty of $0.2 \%$ is assigned due to the different $K_{S}^{0}$ contamination seen in data and $\mathrm{MC}$ for both central and combined tracks. The corresponding effect arising from $\Lambda$ contamination is expected to be negligible.

- The systematic uncertainty on the remaining photoproduction background is estimated to be $30 \%$. This results in an uncertainty on the measured densities up to $0.9 \%$ at small $x$ and $Q^{2}$. At large $x$ and $Q^{2}$ the contribution from photoproduction is small and its uncertainty is negligible.

The dominant systematic uncertainties are the uncertainties arising from the model dependence of the data correction, which are largely correlated, and uncertainties associated with the track reconstruction, which are uncorrelated. The systematic uncertainties shown in the figures and tables are calculated by adding all contributions in quadrature. The total systematic uncertainty for the single differential measurements is below $2.5 \%$ for most analysis bins.

\section{Results}

The measurements of the charged particle densities as a function of pseudorapidity and transverse momentum in the phase space summarised in Table 1 are listed in Tables 2 to 9 and shown in Figs. 3 to 10.

\subsection{Charged particle densities as a function of pseudorapidity}

The charged particle densities as a function of $\eta^{*}$ were measured separately for $p_{T}^{*}<1 \mathrm{GeV}$ and for $1<p_{T}^{*}<10 \mathrm{GeV}$, as shown in Fig. 3. In the soft $p_{T}^{*}$ region, the pseudorapidity distribution is almost flat in the $1.5 \lesssim \eta^{*} \lesssim 3$ range with about 1.7 charged particles per unit of pseudorapidity. The distribution falls at small $\eta^{*}$ due to the cut on pseudorapidity in the laboratory frame. In the hard $p_{T}^{*}$ region the distribution becomes more peaked near $\eta^{*}=2.5$, with a maximum of 0.23 charged particles per unit of pseudorapidity. For $1<p_{T}^{*}<10 \mathrm{GeV}$ the density increases rather strongly up to $\eta^{*} \approx 2.5$, a behaviour expected from the strong ordering of transverse momentum towards the hard scattering vertex.
Table 2 Charged particle densities as a function of $\eta^{*}$ for $0<p_{T}^{*}<$ $1 \mathrm{GeV}$ with relative statistical (stat.) and systematic (sys.) uncertainties given in per cent. The phase space is defined in Table 1

\begin{tabular}{llll}
\hline $0<p_{T}^{*}<1 \mathrm{GeV}$ & & \\
\hline$\eta^{*}$ range & $1 / N \cdot \mathrm{d} n / \mathrm{d} \eta^{*}$ & stat. (\%) & sys. (\%) \\
\hline $0.0-1.0$ & 1.019 & 0.06 & 6.2 \\
$1.0-1.6$ & 1.577 & 0.03 & 3.4 \\
$1.6-2.1$ & 1.717 & 0.03 & 2.7 \\
$2.1-2.6$ & 1.754 & 0.03 & 2.1 \\
$2.6-3.1$ & 1.706 & 0.03 & 1.4 \\
$3.1-3.7$ & 1.467 & 0.04 & 1.4 \\
$3.7-5.0$ & 0.691 & 0.08 & 1.7 \\
\hline
\end{tabular}

Table 3 Charged particle densities as a function of $\eta^{*}$ for $1<p_{T}^{*}<$ $10 \mathrm{GeV}$ with relative statistical (stat.) and systematic (sys.) uncertainties given in per cent. The phase space is defined in Table 1

\begin{tabular}{llll}
\hline \multicolumn{2}{l}{$l<p_{T}^{*}<10 \mathrm{GeV}$} \\
$\eta^{*}$ range & $1 / N \cdot \mathrm{d} n / \mathrm{d} \eta^{*}$ & stat. (\%) & sys. (\%) \\
\hline $0.0-0.5$ & 0.0807 & 0.26 & 6.9 \\
$0.5-1.0$ & 0.1448 & 0.14 & 4.6 \\
$1.0-1.5$ & 0.1835 & 0.11 & 2.4 \\
$1.5-2.0$ & 0.2066 & 0.10 & 1.4 \\
$2.0-2.5$ & 0.2255 & 0.09 & 1.8 \\
$2.5-3.0$ & 0.2251 & 0.09 & 1.9 \\
$3.0-3.7$ & 0.1668 & 0.13 & 2.3 \\
$3.7-5.0$ & 0.0329 & 0.42 & 4.5 \\
\hline
\end{tabular}

Figure 3 also shows the predictions of the DGLAP-like model RAPGAP based on different PDF sets. In the soft $p_{T}^{*}$ region all NLO PDFs (HERAPDF1.0 [13], CTEQ6.6 [83], GRV98NLO [84]) show similar results and predict less particles compared to calculations using the default LO PDF set CTEQ6L(LO). All predictions are close to the data. At large $p_{T}^{*}$, differences between the NLO PDF sets are observed, with CTEQ6L(LO) being closest to the data, although the differences to the data are still larger than the differences between the various PDF predictions. Similar PDF uncertainties are observed when using the CDM model as implemented in DJANGOH.

To check the sensitivity to hadronisation effects, the RAPGAP predictions obtained with three sets of fragmentation parameters are compared to the data in Fig. 4: parameters tuned by ALEPH [57], by the Professor tuning tool [58] and default PYTHIA6.424 fragmentation parameters. Significant differences between these three samples are seen in the soft $p_{T}^{*}$ region, where the data are best described by the ALEPH tune. At large transverse momenta they give similar results but none of them describes the data.

Predictions from models with different approaches for QCD radiation (see Sect. 2) are shown in Fig. 5. The data 
Table 4 Charged particle densities as a function of $\eta^{*}$ for $0<p_{T}^{*}<$ $1 \mathrm{GeV}$ for different $Q^{2}$ and $x$ intervals with relative statistical (stat.) and systematic (sys.) uncertainties given in per cent. The phase space is defined in Table 1

\begin{tabular}{|c|c|c|c|c|}
\hline \multicolumn{5}{|l|}{$0<p_{T}^{*}<1 \mathrm{GeV}$} \\
\hline$Q^{2}, x$ intervals & $\eta^{*}$ range & $1 / N \cdot \mathrm{d} n / \mathrm{d} \eta^{*}$ & stat. $(\%)$ & sys. (\%) \\
\hline \multirow{7}{*}{$\begin{array}{l}5<Q^{2}<10 \mathrm{GeV}^{2} \\
0.0001<x<0.00024\end{array}$} & $0.0-1.0$ & 1.398 & 0.14 & 5.8 \\
\hline & $1.0-1.6$ & 1.621 & 0.09 & 3.5 \\
\hline & $1.6-2.1$ & 1.727 & 0.08 & 2.8 \\
\hline & $2.1-2.6$ & 1.760 & 0.08 & 3.5 \\
\hline & $2.6-3.1$ & 1.749 & 0.08 & 3.5 \\
\hline & $3.1-3.7$ & 1.650 & 0.10 & 2.6 \\
\hline & $3.7-5.0$ & 0.683 & 0.23 & 2.1 \\
\hline \multirow{6}{*}{$\begin{array}{l}5<Q^{2}<10 \mathrm{GeV}^{2} \\
0.00024<x<0.0005\end{array}$} & $0.0-1.5$ & 1.241 & 0.22 & 4.4 \\
\hline & $1.5-2.3$ & 1.682 & 0.12 & 2.7 \\
\hline & $2.3-2.8$ & 1.732 & 0.10 & 3.2 \\
\hline & $2.8-3.3$ & 1.671 & 0.10 & 2.1 \\
\hline & $3.3-3.9$ & 1.347 & 0.12 & 2.0 \\
\hline & $3.9-5.0$ & 0.652 & 0.24 & 2.1 \\
\hline \multirow{4}{*}{$\begin{array}{l}5<Q^{2}<10 \mathrm{GeV}^{2} \\
0.0005<x<0.002\end{array}$} & $0.5-2.0$ & 1.288 & 0.21 & 4.4 \\
\hline & $2.0-2.9$ & 1.613 & 0.13 & 1.8 \\
\hline & $2.9-3.7$ & 1.272 & 0.15 & 2.0 \\
\hline & $3.7-5.0$ & 0.554 & 0.28 & 2.5 \\
\hline \multirow{7}{*}{$\begin{array}{l}10<Q^{2}<20 \mathrm{GeV}^{2} \\
0.0002<x<0.00052\end{array}$} & $0.0-1.0$ & 1.464 & 0.15 & 5.9 \\
\hline & $1.0-1.6$ & 1.721 & 0.10 & 2.5 \\
\hline & $1.6-2.1$ & 1.820 & 0.09 & 2.6 \\
\hline & $2.1-2.6$ & 1.865 & 0.09 & 2.8 \\
\hline & $2.6-3.1$ & 1.857 & 0.09 & 2.1 \\
\hline & $3.1-3.7$ & 1.680 & 0.10 & 1.6 \\
\hline & $3.7-5.0$ & 0.784 & 0.23 & 1.7 \\
\hline \multirow{6}{*}{$\begin{array}{l}10<Q^{2}<20 \mathrm{GeV}^{2} \\
0.00052<x<0.0011\end{array}$} & $0.0-1.5$ & 1.268 & 0.22 & 5.2 \\
\hline & $1.5-2.3$ & 1.790 & 0.12 & 2.6 \\
\hline & $2.3-2.8$ & 1.819 & 0.10 & 2.1 \\
\hline & $2.8-3.3$ & 1.685 & 0.10 & 1.7 \\
\hline & $3.3-3.9$ & 1.312 & 0.12 & 1.9 \\
\hline & $3.9-5.0$ & 0.700 & 0.23 & 2.0 \\
\hline \multirow{4}{*}{$\begin{array}{l}10<Q^{2}<20 \mathrm{GeV}^{2} \\
0.0011<x<0.0037\end{array}$} & $0.5-2.0$ & 1.34 & 0.20 & 8.6 \\
\hline & $2.0-2.9$ & 1.650 & 0.13 & 1.9 \\
\hline & $2.9-3.7$ & 1.263 & 0.14 & 2.3 \\
\hline & $3.7-5.0$ & 0.550 & 0.28 & 3.4 \\
\hline \multirow{7}{*}{$\begin{array}{l}20<Q^{2}<100 \mathrm{GeV}^{2} \\
0.0004<x<0.0017\end{array}$} & $0.0-1.0$ & 1.461 & 0.15 & 5.8 \\
\hline & $1.0-1.6$ & 1.820 & 0.09 & 2.7 \\
\hline & $1.6-2.1$ & 1.928 & 0.08 & 2.4 \\
\hline & $2.1-2.6$ & 1.951 & 0.08 & 2.4 \\
\hline & $2.6-3.1$ & 1.883 & 0.08 & 2.2 \\
\hline & $3.1-3.7$ & 1.601 & 0.10 & 1.7 \\
\hline & $3.7-5.0$ & 0.883 & 0.21 & 2.1 \\
\hline
\end{tabular}

Table 4 (Continued)

\begin{tabular}{lllll}
\hline $0<p_{T}^{*}<1 \mathrm{GeV}$ & & & & \\
\hline$Q^{2}, x$ intervals & $\eta^{*}$ range & $1 / N \cdot \mathrm{d} n / \mathrm{d} \eta^{*}$ & stat. (\%) & sys. (\%) \\
\hline $20<Q^{2}<100 \mathrm{GeV}^{2}$ & $0.0-1.5$ & 1.077 & 0.20 & 5.4 \\
$0.0017<x<0.01$ & $1.5-2.2$ & 1.783 & 0.09 & 2.6 \\
& $2.2-2.9$ & 1.714 & 0.09 & 1.8 \\
& $2.9-3.7$ & 1.445 & 0.11 & 1.9 \\
& $3.7-5.0$ & 0.634 & 0.22 & 2.0 \\
\hline
\end{tabular}

Table 5 Charged particle densities as a function of $\eta^{*}$ for $1<p_{T}^{*}<$ $10 \mathrm{GeV}$ for different $Q^{2}$ and $x$ intervals with relative statistical (stat.) and systematic (sys.) uncertainties given in per cent. The phase space is defined in Table 1

\begin{tabular}{|c|c|c|c|c|}
\hline \multicolumn{5}{|l|}{$1<p_{T}^{*}<10 \mathrm{GeV}$} \\
\hline$Q^{2}, x$ intervals & $\eta^{*}$ range & $1 / N \cdot \mathrm{d} n / \mathrm{d} \eta^{*}$ & stat. (\%) & sys. (\%) \\
\hline \multirow{8}{*}{$\begin{array}{l}5<Q^{2}<10 \mathrm{GeV}^{2} \\
0.0001<x<0.00024\end{array}$} & $0.0-0.5$ & 0.1365 & 0.39 & 5.7 \\
\hline & $0.5-1.0$ & 0.1551 & 0.29 & 3.5 \\
\hline & $1.0-1.5$ & 0.1679 & 0.27 & 2.3 \\
\hline & $1.5-2.0$ & 0.1818 & 0.26 & 3.5 \\
\hline & $2.0-2.5$ & 0.1961 & 0.26 & 2.4 \\
\hline & $2.5-3.0$ & 0.2084 & 0.24 & 2.4 \\
\hline & $3.0-3.7$ & 0.1984 & 0.31 & 2.3 \\
\hline & $3.7-5.0$ & 0.0501 & 1.02 & 3.6 \\
\hline \multirow{7}{*}{$\begin{array}{l}5<Q^{2}<10 \mathrm{GeV}^{2} \\
0.00024<x<0.0005\end{array}$} & $0.0-1.0$ & 0.1115 & 0.70 & 7.3 \\
\hline & $1.0-1.5$ & 0.1575 & 0.33 & 3.4 \\
\hline & $1.5-2.0$ & 0.1758 & 0.31 & 3.3 \\
\hline & $2.0-2.5$ & 0.1903 & 0.30 & 5.2 \\
\hline & $2.5-3.0$ & 0.2037 & 0.29 & 1.9 \\
\hline & $3.0-3.7$ & 0.1626 & 0.38 & 2.6 \\
\hline & $3.7-5.0$ & 0.0311 & 1.33 & 8.0 \\
\hline \multirow{6}{*}{$\begin{array}{l}5<Q^{2}<10 \mathrm{GeV}^{2} \\
0.0005<x<0.002\end{array}$} & $0.5-1.5$ & 0.1244 & 0.66 & 4.5 \\
\hline & $1.5-2.0$ & 0.1675 & 0.32 & 4.7 \\
\hline & $2.0-2.5$ & 0.1811 & 0.31 & 3.0 \\
\hline & $2.5-3.0$ & 0.1686 & 0.32 & 2.3 \\
\hline & $3.0-3.7$ & 0.0978 & 0.51 & 3.0 \\
\hline & $3.7-5.0$ & 0.01071 & 2.21 & 4.7 \\
\hline \multirow{8}{*}{$\begin{array}{l}10<Q^{2}<20 \mathrm{GeV}^{2} \\
0.0002<x<0.00052\end{array}$} & $0.0-0.5$ & 0.1506 & 0.42 & 6.1 \\
\hline & $0.5-1.0$ & 0.1764 & 0.30 & 2.1 \\
\hline & $1.0-1.5$ & 0.1959 & 0.28 & 2.7 \\
\hline & $1.5-2.0$ & 0.2180 & 0.26 & 1.7 \\
\hline & $2.0-2.5$ & 0.2444 & 0.25 & 1.9 \\
\hline & $2.5-3.0$ & 0.2646 & 0.23 & 2.7 \\
\hline & $3.0-3.7$ & 0.2334 & 0.31 & 1.4 \\
\hline & $3.7-5.0$ & 0.0552 & 1.03 & 2.7 \\
\hline
\end{tabular}


Table 5 (Continued)

\begin{tabular}{|c|c|c|c|c|}
\hline \multicolumn{5}{|l|}{$1<p_{T}^{*}<10 \mathrm{GeV}$} \\
\hline$Q^{2}, x$ intervals & $\eta^{*}$ range & $1 / N \cdot \mathrm{d} n / \mathrm{d} \eta^{*}$ & stat. $(\%)$ & sys. (\%) \\
\hline \multirow{7}{*}{$\begin{array}{l}10<Q^{2}<20 \mathrm{GeV}^{2} \\
0.00052<x<0.0011\end{array}$} & $0.0-1.0$ & 0.123 & 0.69 & 8.3 \\
\hline & $1.0-1.5$ & 0.1910 & 0.30 & 3.4 \\
\hline & $1.5-2.0$ & 0.2145 & 0.27 & 3.1 \\
\hline & $2.0-2.5$ & 0.2409 & 0.26 & 3.6 \\
\hline & $2.5-3.0$ & 0.2415 & 0.26 & 2.6 \\
\hline & $3.0-3.7$ & 0.1750 & 0.37 & 2.4 \\
\hline & $3.7-5.0$ & 0.0315 & 1.34 & 3.7 \\
\hline \multirow{6}{*}{$\begin{array}{l}10<Q^{2}<20 \mathrm{GeV}^{2} \\
0.0011<x<0.0037\end{array}$} & $0.5-1.5$ & 0.1496 & 0.58 & 6.6 \\
\hline & $1.5-2.0$ & 0.2086 & 0.27 & 3.8 \\
\hline & $2.0-2.5$ & 0.2246 & 0.26 & 4.5 \\
\hline & $2.5-3.0$ & 0.1997 & 0.28 & 3.8 \\
\hline & $3.0-3.7$ & 0.1051 & 0.48 & 3.8 \\
\hline & $3.7-5.0$ & 0.01028 & 2.38 & 7.7 \\
\hline \multirow{8}{*}{$\begin{array}{l}20<Q^{2}<100 \mathrm{GeV}^{2} \\
0.0004<x<0.0017\end{array}$} & $0.0-0.5$ & 0.1581 & 0.42 & 6.1 \\
\hline & $0.5-1.0$ & 0.2105 & 0.27 & 2.3 \\
\hline & $1.0-1.5$ & 0.2479 & 0.23 & 2.5 \\
\hline & $1.5-2.0$ & 0.2820 & 0.22 & 2.1 \\
\hline & $2.0-2.5$ & 0.3188 & 0.20 & 2.3 \\
\hline & $2.5-3.0$ & 0.3386 & 0.20 & 1.4 \\
\hline & $3.0-3.7$ & 0.2601 & 0.28 & 1.5 \\
\hline & $3.7-5.0$ & 0.0602 & 0.95 & 1.8 \\
\hline \multirow{7}{*}{$\begin{array}{l}20<Q^{2}<100 \mathrm{GeV}^{2} \\
0.0017<x<0.01\end{array}$} & $0.0-1.0$ & 0.118 & 0.63 & 9.0 \\
\hline & $1.0-1.5$ & 0.251 & 0.22 & 4.5 \\
\hline & $1.5-2.0$ & 0.2966 & 0.19 & 3.4 \\
\hline & $2.0-2.5$ & 0.3167 & 0.19 & 2.1 \\
\hline & $2.5-3.0$ & 0.2799 & 0.20 & 2.1 \\
\hline & $3.0-3.7$ & 0.1587 & 0.34 & 2.4 \\
\hline & $3.7-5.0$ & 0.02112 & 1.49 & 4.6 \\
\hline
\end{tabular}

Table 6 Charged particle densities as a function as a function of $p_{T}^{*}$ in the region $0<\eta^{*}<1.5$ shown with relative statistical (stat.) and systematic (sys.) uncertainties given in per cent. The phase space is defined in Table 1

\begin{tabular}{llll}
\hline $0<\eta^{*}<1.5$ & & & \\
\hline$p_{T}^{*}$ range $[\mathrm{GeV}]$ & $1 / N \cdot \mathrm{d} n / \mathrm{d} p_{T}^{*}\left[\mathrm{GeV}^{-1}\right]$ & stat. $(\%)$ & sys. $(\%)$ \\
\hline $0.2-0.4$ & 3.952 & 0.01 & 2.0 \\
$0.4-0.6$ & 2.431 & 0.02 & 1.6 \\
$0.6-1.0$ & 0.954 & 0.04 & 1.8 \\
$1.0-2.0$ & 0.1686 & 0.15 & 2.5 \\
$2.0-4.0$ & $1.549 \cdot 10^{-2}$ & 0.70 & 2.0 \\
$4.0-10.0$ & $7.15 \cdot 10^{-4}$ & 5.41 & 1.9 \\
\hline
\end{tabular}

Table 7 Charged particle densities as a function as a function of $p_{T}^{*}$ in the region $1.5<\eta^{*}<5$ shown with relative statistical (stat.) and systematic (sys.) uncertainties given in per cent. The phase space is defined in Table 1

\begin{tabular}{llll}
\hline $1.5<\eta^{*}<5$ & & & \\
\hline$p_{T}^{*}$ range $[\mathrm{GeV}]$ & $1 / N \cdot \mathrm{d} n / \mathrm{d} p_{T}^{*}\left[\mathrm{GeV}^{-1}\right]$ & stat. $(\%)$ & sys. (\%) \\
\hline $0.0-0.3$ & 5.24 & 0.01 & 1.8 \\
$0.3-0.6$ & 6.10 & 0.01 & 1.7 \\
$0.6-1.0$ & 2.234 & 0.02 & 1.8 \\
$1.0-1.5$ & 0.6193 & 0.05 & 1.5 \\
$1.5-2.1$ & 0.1849 & 0.10 & 1.5 \\
$2.1-3.0$ & $5.23 \cdot 10^{-2}$ & 0.23 & 2.0 \\
$3.0-4.0$ & $1.381 \cdot 10^{-2}$ & 0.47 & 2.0 \\
$4.0-5.0$ & $4.14 \cdot 10^{-3}$ & 0.84 & 2.4 \\
$5.0-6.3$ & $1.402 \cdot 10^{-3}$ & 1.67 & 2.8 \\
$6.3-7.9$ & $3.98 \cdot 10^{-4}$ & 3.47 & 2.5 \\
$7.9-10.0$ & $1.061 \cdot 10^{-4}$ & 7.60 & 3.2 \\
\hline
\end{tabular}

Table 8 Charged particle densities as a function of $p_{T}^{*}$ in the region $0<\eta^{*}<1.5$ for different $Q^{2}$ and $x$ intervals shown with relative statistical (stat.) and systematic (sys.) uncertainties given in per cent. The phase space is defined in Table 1

\begin{tabular}{|c|c|c|c|c|}
\hline \multicolumn{5}{|l|}{$0<\eta^{*}<1.5$} \\
\hline$Q^{2}, x$ intervals & $\begin{array}{l}p_{T}^{*} \text { range } \\
{[\mathrm{GeV}]}\end{array}$ & $\begin{array}{l}1 / N \cdot \mathrm{d} n / \mathrm{d} p_{T}^{*} \\
{\left[\mathrm{GeV}^{-1}\right]}\end{array}$ & $\begin{array}{l}\text { stat. } \\
(\%)\end{array}$ & $\begin{array}{l}\text { sys } \\
(\%\end{array}$ \\
\hline \multirow{6}{*}{$\begin{array}{l}5<Q^{2}<10 \mathrm{GeV}^{2} \\
0.0001<x<0.00024\end{array}$} & $0.2-0.4$ & 4.76 & 0.03 & 3.2 \\
\hline & $0.4-0.6$ & 2.92 & 0.04 & 2.9 \\
\hline & $0.6-1.0$ & 1.144 & 0.10 & 3.7 \\
\hline & $1.0-2.0$ & 0.1955 & 0.39 & 3.0 \\
\hline & $2.0-4.0$ & $1.489 \cdot 10^{-2}$ & 1.89 & 3.2 \\
\hline & $4.0-10.0$ & $5.69 \cdot 10^{-4}$ & 15.36 & 6.0 \\
\hline \multirow{6}{*}{$\begin{array}{l}5<Q^{2}<10 \mathrm{GeV}^{2} \\
0.00024<x<0.0005\end{array}$} & $0.2-0.4$ & 3.99 & 0.05 & 2.5 \\
\hline & $0.4-0.6$ & 2.53 & 0.06 & 2.6 \\
\hline & $0.6-1.0$ & 0.994 & 0.14 & 2.9 \\
\hline & $1.0-2.0$ & 0.1611 & 0.52 & 3.3 \\
\hline & $2.0-4.0$ & $1.286 \cdot 10^{-2}$ & 2.58 & 2.7 \\
\hline & $4.0-10.0$ & $5.1 \cdot 10^{-4}$ & 21.40 & 4.9 \\
\hline \multirow{5}{*}{$\begin{array}{l}5<Q^{2}<10 \mathrm{GeV}^{2} \\
0.0005<x<0.002\end{array}$} & $0.2-0.6$ & 2.097 & 0.11 & 3.0 \\
\hline & $0.6-1.0$ & 0.659 & 0.19 & 3.4 \\
\hline & $1.0-2.0$ & 0.1113 & 0.74 & 2.8 \\
\hline & $2.0-4.0$ & $8.82 \cdot 10^{-3}$ & 3.40 & 3.1 \\
\hline & $4.0-10.0$ & $3.3 \cdot 10^{-4}$ & 34.47 & 5.5 \\
\hline \multirow{6}{*}{$\begin{array}{l}10<Q^{2}<20 \mathrm{GeV}^{2} \\
0.0002<x<0.00052\end{array}$} & $0.2-0.4$ & 4.97 & 0.04 & 2.5 \\
\hline & $0.4-0.6$ & 3.060 & 0.05 & 2.7 \\
\hline & $0.6-1.0$ & 1.229 & 0.11 & 2.6 \\
\hline & $1.0-2.0$ & 0.2155 & 0.40 & 3.5 \\
\hline & $2.0-4.0$ & $1.960 \cdot 10^{-2}$ & 1.87 & 3.1 \\
\hline & $4.0-10.0$ & $9.2 \cdot 10^{-4}$ & 14.13 & 3.3 \\
\hline
\end{tabular}


Table 8 (Continued)

\begin{tabular}{|c|c|c|c|c|}
\hline \multicolumn{5}{|l|}{$0<\eta^{*}<1.5$} \\
\hline$\overline{Q^{2}, x \text { intervals }}$ & $\begin{array}{l}p_{T}^{*} \text { range } \\
{[\mathrm{GeV}]}\end{array}$ & $\begin{array}{l}1 / N \cdot \mathrm{d} n / \mathrm{d} p_{T}^{*} \\
{\left[\mathrm{GeV}^{-1}\right]}\end{array}$ & $\begin{array}{l}\text { stat. } \\
(\%)\end{array}$ & $\begin{array}{l}\text { sys } \\
(\%)\end{array}$ \\
\hline \multirow{6}{*}{$\begin{array}{l}10<Q^{2}<20 \mathrm{GeV}^{2} \\
0.00052<x<0.0011\end{array}$} & $0.2-0.4$ & 4.05 & 0.05 & 3.0 \\
\hline & $0.4-0.6$ & 2.593 & 0.06 & 2.9 \\
\hline & $0.6-1.0$ & 1.033 & 0.13 & 3.5 \\
\hline & $1.0-2.0$ & 0.1811 & 0.50 & 4.2 \\
\hline & $2.0-4.0$ & $1.623 \cdot 10^{-2}$ & 2.34 & 5.8 \\
\hline & $4.0-10.0$ & $7.5 \cdot 10^{-4}$ & 18.91 & 3.2 \\
\hline \multirow{5}{*}{$\begin{array}{l}10<Q^{2}<20 \mathrm{GeV}^{2} \\
0.0011<x<0.0037\end{array}$} & $0.2-0.6$ & 2.124 & 0.10 & 4.5 \\
\hline & $0.6-1.0$ & 0.692 & 0.18 & 4.1 \\
\hline & $1.0-2.0$ & 0.129 & 0.66 & 4.8 \\
\hline & $2.0-4.0$ & $1.119 \cdot 10^{-2}$ & 3.12 & 3.0 \\
\hline & $4.0-10.0$ & $4.42 \cdot 10^{-4}$ & 28.92 & 6.0 \\
\hline \multirow{6}{*}{$\begin{array}{l}20<Q^{2}<100 \mathrm{GeV}^{2} \\
0.0004<x<0.0017\end{array}$} & $0.2-0.4$ & 5.00 & 0.04 & 2.5 \\
\hline & $0.4-0.6$ & 3.156 & 0.04 & 2.4 \\
\hline & $0.6-1.0$ & 1.296 & 0.97 & 2.8 \\
\hline & $1.0-2.0$ & 0.2474 & 0.36 & 3.7 \\
\hline & $2.0-4.0$ & $2.579 \cdot 10^{-2}$ & 1.57 & 4.7 \\
\hline & $4.0-10.0$ & $1.40 \cdot 10^{-3}$ & 11.28 & 2.8 \\
\hline \multirow{6}{*}{$\begin{array}{l}20<Q^{2}<100 \mathrm{GeV}^{2} \\
0.0017<x<0.01\end{array}$} & $0.2-0.4$ & 3.356 & 0.05 & 2.8 \\
\hline & $0.4-0.6$ & 2.254 & 0.05 & 2.8 \\
\hline & $0.6-1.0$ & 0.945 & 0.12 & 3.6 \\
\hline & $1.0-2.0$ & 0.1905 & 0.42 & 5.4 \\
\hline & $2.0-4.0$ & $2.185 \cdot 10^{-2}$ & 1.74 & 6.9 \\
\hline & $4.0-10.0$ & $1.21 \cdot 10^{-3}$ & 13.18 & 3.5 \\
\hline
\end{tabular}

are compared to the CDM model DJANGOH, the DGLAPbased MC RAPGAP and Herwig++ and the CCFM model CASCADE. In the soft $p_{T}^{*}$ region, DJANGOH and RAPGAP describe the data within the PDF uncertainties (Fig. 3). Herwig++, which uses the cluster fragmentation model, provides a reasonable description of the data in the central region. The effect of not using the POWHEG option in Herwig++ also has been investigated. Only small differences were observed which are not considered further in this paper. CASCADE predicts too high multiplicities in most of the measured $\eta^{*}$ range. In the region of $1<p_{T}^{*}<10 \mathrm{GeV}$ the best description of the data is achieved by DJANGOH. RAPGAP strongly undershoots the data in the central region. Herwig++ predicts a spectrum which is even below the prediction of RAPGAP. CASCADE is significantly above the data in a wide range of $\eta^{*}$.

The charged particle densities as a function of $\eta^{*}$ are shown in Fig. 6 for $p_{T}^{*}<1 \mathrm{GeV}$ in eight $\left(x, Q^{2}\right)$ bins. The data are compared to predictions of the DJANGOH, RAPGAP, Herwig++ and CASCADE generators. DJANGOH provides a good description of the data over the full kinematic
Table 9 Charged particle densities as a function of $p_{T}^{*}$ in the region $1.5<\eta^{*}<5$ for different $Q^{2}$ and $x$ intervals shown with relative statistical (stat.) and systematic (sys.) uncertainties given in per cent. The phase space is defined in Table 1

\begin{tabular}{|c|c|c|c|c|}
\hline \multicolumn{5}{|l|}{$1.5<\eta^{*}<5$} \\
\hline$Q^{2}, x$ intervals & $\begin{array}{l}p_{T}^{*} \text { range } \\
{[\mathrm{GeV}]}\end{array}$ & $\begin{array}{l}1 / N \cdot \mathrm{d} n / \mathrm{d} p_{T}^{*} \\
{\left[\mathrm{GeV}^{-1}\right]}\end{array}$ & $\begin{array}{l}\text { stat. } \\
(\%)\end{array}$ & $\begin{array}{l}\text { sys. } \\
(\%)\end{array}$ \\
\hline \multirow{11}{*}{$\begin{array}{l}5<Q^{2}<10 \mathrm{GeV}^{2} \\
0.0001<x<0.00024\end{array}$} & $0.0-0.3$ & 5.60 & 0.04 & 2.5 \\
\hline & $0.3-0.6$ & 6.83 & 0.04 & 2.8 \\
\hline & $0.6-1.0$ & 2.290 & 0.07 & 3.6 \\
\hline & $1.0-1.5$ & 0.639 & 0.14 & 3.3 \\
\hline & $1.5-2.1$ & 0.1897 & 0.30 & 2.7 \\
\hline & $2.1-3.0$ & $5.12 \cdot 10^{-2}$ & 0.69 & 4.0 \\
\hline & $3.0-4.0$ & $1.378 \cdot 10^{-2}$ & 1.40 & 4.8 \\
\hline & $4.0-5.0$ & $3.97 \cdot 10^{-3}$ & 2.46 & 8.6 \\
\hline & $5.0-6.3$ & $1.40 \cdot 10^{-3}$ & 4.68 & 9.7 \\
\hline & $6.3-7.9$ & $4.34 \cdot 10^{-4}$ & 9.33 & 6.2 \\
\hline & $7.9-10.0$ & $9.3 \cdot 10^{-5}$ & 20.80 & 13.2 \\
\hline \multirow{11}{*}{$\begin{array}{l}5<Q^{2}<10 \mathrm{GeV}^{2} \\
0.00024<x<0.0005\end{array}$} & $0.0-0.3$ & 5.57 & 0.04 & 2.8 \\
\hline & $0.3-0.6$ & 6.64 & 0.04 & 2.6 \\
\hline & $0.6-1.0$ & 2.207 & 0.08 & 2.2 \\
\hline & $1.0-1.5$ & 0.587 & 0.17 & 1.7 \\
\hline & $1.5-2.1$ & 0.161 & 0.36 & 1.8 \\
\hline & $2.1-3.0$ & $4.12 \cdot 10^{-2}$ & 0.85 & 3.2 \\
\hline & $3.0-4.0$ & $1.002 \cdot 10^{-2}$ & 1.83 & 5.7 \\
\hline & $4.0-5.0$ & $2.63 \cdot 10^{-3}$ & 3.46 & 6.7 \\
\hline & $5.0-6.3$ & $7.69 \cdot 10^{-4}$ & 7.08 & 5.7 \\
\hline & $6.3-7.9$ & $2.46 \cdot 10^{-4}$ & 13.98 & 10.4 \\
\hline & $7.9-10.0$ & $6.65 \cdot 10^{-5}$ & 32.90 & 18.6 \\
\hline \multirow{10}{*}{$\begin{array}{l}5<Q^{2}<10 \mathrm{GeV}^{2} \\
0.0005<x<0.002\end{array}$} & $0.0-0.3$ & 4.96 & 0.05 & 2.2 \\
\hline & $0.3-0.6$ & 5.74 & 0.04 & 2.1 \\
\hline & $0.6-1.0$ & 1.863 & 0.08 & 3.4 \\
\hline & $1.0-1.5$ & 0.472 & 0.19 & 2.4 \\
\hline & $1.5-2.1$ & 0.1214 & 0.40 & 2.5 \\
\hline & $2.1-3.0$ & $2.85 \cdot 10^{-2}$ & 1.02 & 5.5 \\
\hline & $3.0-4.0$ & $6.14 \cdot 10^{-3}$ & 2.40 & 6.1 \\
\hline & $4.0-5.0$ & $1.55 \cdot 10^{-3}$ & 4.94 & 5.4 \\
\hline & $5.0-6.3$ & $4.401 \cdot 10^{-4}$ & 10.49 & 12.8 \\
\hline & $6.3-7.9$ & $1.11 \cdot 10^{-4}$ & 24.17 & 13.6 \\
\hline \multirow{11}{*}{$\begin{array}{l}10<Q^{2}<20 \mathrm{GeV}^{2} \\
0.0002<x<0.00052\end{array}$} & $0.0-0.3$ & 5.975 & 0.04 & 1.5 \\
\hline & $0.3-0.6$ & 7.16 & 0.04 & 2.0 \\
\hline & $0.6-1.0$ & 2.517 & 0.07 & 1.7 \\
\hline & $1.0-1.5$ & 0.742 & 0.15 & 2.4 \\
\hline & $1.5-2.1$ & 0.2317 & 0.29 & 2.3 \\
\hline & $2.1-3.0$ & $6.82 \cdot 10^{-2}$ & 0.64 & 3.4 \\
\hline & $3.0-4.0$ & $1.848 \cdot 10^{-2}$ & 1.30 & 3.6 \\
\hline & $4.0-5.0$ & $5.83 \cdot 10^{-3}$ & 2.27 & 5.2 \\
\hline & $5.0-6.3$ & $2.00 \cdot 10^{-3}$ & 4.45 & 2.5 \\
\hline & $6.3-7.9$ & $5.88 \cdot 10^{-4}$ & 8.91 & 2.9 \\
\hline & $7.9-10.0$ & $1.942 \cdot 10^{-4}$ & 17.77 & 5.3 \\
\hline
\end{tabular}


Table 9 (Continued)

\begin{tabular}{|c|c|c|c|c|}
\hline \multicolumn{5}{|l|}{$1.5<\eta^{*}<5$} \\
\hline$Q^{2}, x$ intervals & $\begin{array}{l}p_{T}^{*} \text { range } \\
{[\mathrm{GeV}]}\end{array}$ & $\begin{array}{l}1 / N \cdot \mathrm{d} n / \mathrm{d} p_{T}^{*} \\
{\left[\mathrm{GeV}^{-1}\right]}\end{array}$ & $\begin{array}{l}\text { stat. } \\
(\%)\end{array}$ & $\begin{array}{l}\text { sys. } \\
(\%)\end{array}$ \\
\hline \multirow{11}{*}{$\begin{array}{l}10<Q^{2}<20 \mathrm{GeV}^{2} \\
0.00052<x<0.0011\end{array}$} & $0.0-0.3$ & 5.824 & 0.05 & 1.5 \\
\hline & $0.3-0.6$ & 6.77 & 0.04 & 1.7 \\
\hline & $0.6-1.0$ & 2.332 & 0.08 & 2.2 \\
\hline & $1.0-1.5$ & 0.657 & 0.16 & 1.8 \\
\hline & $1.5-2.1$ & 0.1948 & 0.31 & 2.3 \\
\hline & $2.1-3.0$ & 0.0538 & 0.74 & 3.0 \\
\hline & $3.0-4.0$ & $1.297 \cdot 10^{-2}$ & 1.56 & 5.9 \\
\hline & $4.0-5.0$ & $3.94 \cdot 10^{-3}$ & 2.90 & 4.5 \\
\hline & $5.0-6.3$ & $1.33 \cdot 10^{-3}$ & 5.80 & 6.9 \\
\hline & $6.3-7.9$ & $3.40 \cdot 10^{-4}$ & 12.91 & 6.3 \\
\hline & $7.9-10.0$ & $7.7 \cdot 10^{-5}$ & 30.96 & 8.6 \\
\hline \multirow{10}{*}{$\begin{array}{l}10<Q^{2}<20 \mathrm{GeV}^{2} \\
0.0011<x<0.0037\end{array}$} & $0.0-0.3$ & 5.12 & 0.05 & 2.2 \\
\hline & $0.3-0.6$ & 5.65 & 0.04 & 2.4 \\
\hline & $0.6-1.0$ & 1.946 & 0.08 & 2.0 \\
\hline & $1.0-1.5$ & 0.538 & 0.17 & 2.2 \\
\hline & $1.5-2.1$ & 0.1481 & 0.35 & 3.9 \\
\hline & $2.1-3.0$ & $3.89 \cdot 10^{-2}$ & 0.85 & 3.6 \\
\hline & $3.0-4.0$ & $8.643 \cdot 10^{-3}$ & 1.94 & 3.4 \\
\hline & $4.0-5.0$ & $2.10 \cdot 10^{-3}$ & 3.93 & 4.7 \\
\hline & $5.0-6.3$ & $6.43 \cdot 10^{-4}$ & 8.90 & 12.9 \\
\hline & $6.3-10.0$ & $7.7 \cdot 10^{-5}$ & 46.70 & 10.6 \\
\hline \multirow{11}{*}{$\begin{array}{l}20<Q^{2}<100 \mathrm{GeV}^{2} \\
0.0004<x<0.0017\end{array}$} & $0.0-0.3$ & 6.25 & 0.04 & 1.6 \\
\hline & $0.3-0.6$ & 7.32 & 0.04 & 1.7 \\
\hline & $0.6-1.0$ & 2.673 & 0.07 & 2.1 \\
\hline & $1.0-1.5$ & 0.851 & 0.13 & 2.5 \\
\hline & $1.5-2.1$ & 0.2898 & 0.24 & 2.4 \\
\hline & $2.1-3.0$ & $9.21 \cdot 10^{-2}$ & 0.52 & 2.9 \\
\hline & $3.0-4.0$ & $2.790 \cdot 10^{-2}$ & 1.01 & 2.2 \\
\hline & $4.0-5.0$ & $9.30 \cdot 10^{-3}$ & 1.75 & 3.0 \\
\hline & $5.0-6.3$ & $3.28 \cdot 10^{-3}$ & 3.37 & 3.4 \\
\hline & $6.3-7.9$ & $9.64 \cdot 10^{-4}$ & 6.79 & 4.2 \\
\hline & $7.9-10.0$ & $3.06 \cdot 10^{-4}$ & 14.41 & 3.9 \\
\hline \multirow{11}{*}{$\begin{array}{l}20<Q^{2}<100 \mathrm{GeV}^{2} \\
0.0017<x<0.01\end{array}$} & $0.0-0.3$ & 5.652 & 0.04 & 1.5 \\
\hline & $0.3-0.6$ & 6.052 & 0.04 & 1.6 \\
\hline & $0.6-1.0$ & 2.244 & 0.06 & 1.7 \\
\hline & $1.0-1.5$ & 0.697 & 0.13 & 1.8 \\
\hline & $1.5-2.1$ & 0.2323 & 0.24 & 1.9 \\
\hline & $2.1-3.0$ & $7.12 \cdot 10^{-2}$ & 0.52 & 2.1 \\
\hline & $3.0-4.0$ & $2.025 \cdot 10^{-2}$ & 1.05 & 1.9 \\
\hline & $4.0-5.0$ & $6.32 \cdot 10^{-3}$ & 1.92 & 3.9 \\
\hline & $5.0-6.3$ & $2.20 \cdot 10^{-3}$ & 3.87 & 4.0 \\
\hline & $6.3-7.9$ & $5.91 \cdot 10^{-4}$ & 8.42 & 6.0 \\
\hline & $7.9-10.0$ & $1.38 \cdot 10^{-4}$ & 19.37 & 20.1 \\
\hline
\end{tabular}

range. In general the description of the data by RAPGAP is somewhat worse, with overshooting the data by about $10 \%$ at low $x$. Herwig++ predicts smaller charged particle densities than observed in data in most of the phase space with differences of the order of $10 \%$ at the highest $Q^{2}$. CASCADE is significantly above the data for $\eta^{*}<3$ in all $\left(x, Q^{2}\right)$ bins.

In Fig. 7 the charged particle densities as a function of $\eta^{*}$ are shown in $\left(x, Q^{2}\right)$ bins for $1<p_{T}^{*}<10 \mathrm{GeV}$. The shape of the distributions changes with $x$ and $Q^{2}$ more strongly than what is observed for $p_{T}^{*}<1 \mathrm{GeV}$ (Fig. 6). At small values of $x$ and $Q^{2}$ the measured distribution is less dependent on $\eta^{*}$ compared to the region at high $x$ and $Q^{2}$. None of the models describes all aspects of the data. In general DJANGOH is closest to the data. However it fails to describe the data at low and medium $x$ in the central pseudorapidity region, with downwards deviations of the order of $20 \%$. The RAPGAP prediction is below the data, with the strongest deviation observed at small $x$ and small $\eta^{*}$. Herwig++ significantly undershoots the data. The prediction of CASCADE agrees reasonably well with the measurement at low $x$ and $Q^{2}$, but overshoots the data significantly as $x$ or $Q^{2}$ increases.

\subsection{Charged particle densities as a function of transverse momentum}

In Fig. 8 the charged particle densities as a function of $p_{T}^{*}$ are shown for two pseudorapidity intervals, $0<\eta^{*}<1.5$ (central) and $1.5<\eta^{*}<5$ (current). The shapes of the measured $p_{T}^{*}$ distributions in the two pseudorapidity ranges are similar. The spectrum falls by more than four orders of magnitude from $p_{T}^{*}<1 \mathrm{GeV}$ to $p_{T}^{*} \approx 8 \mathrm{GeV}$. The measurements are compared to the predictions of the DJANGOH, RAPGAP, Herwig++ and CASCADE generators. DJANGOH provides in general a good description of the data, while only at high $p_{T}^{*}$ in the current region deviations from the measurement are observed. The other models fail to describe the data, with the strongest deviations being observed in the central region. The ratio of RAPGAP to data shows a sharp drop at $p_{T}^{*} \approx 1 \mathrm{GeV}$. The $p_{T}^{*}$ spectra predicted by Herwig++ are even softer than those predicted by RAPGAP. CASCADE in general produces higher particle densities than measured.

In Figs. 9 and 10 the charged particle densities as a function of $p_{T}^{*}$ are shown for eight $\left(x, Q^{2}\right)$ bins for the central and the current region, respectively. In the central region the measurement shows a dependence on $x$, such that the number of soft particles is decreasing with increasing $x$ for fixed $Q^{2}$. In the current region this effect is less pronounced. The DJANGOH model provides in general a good description of the data over the full kinematic range in both pseudorapidity 

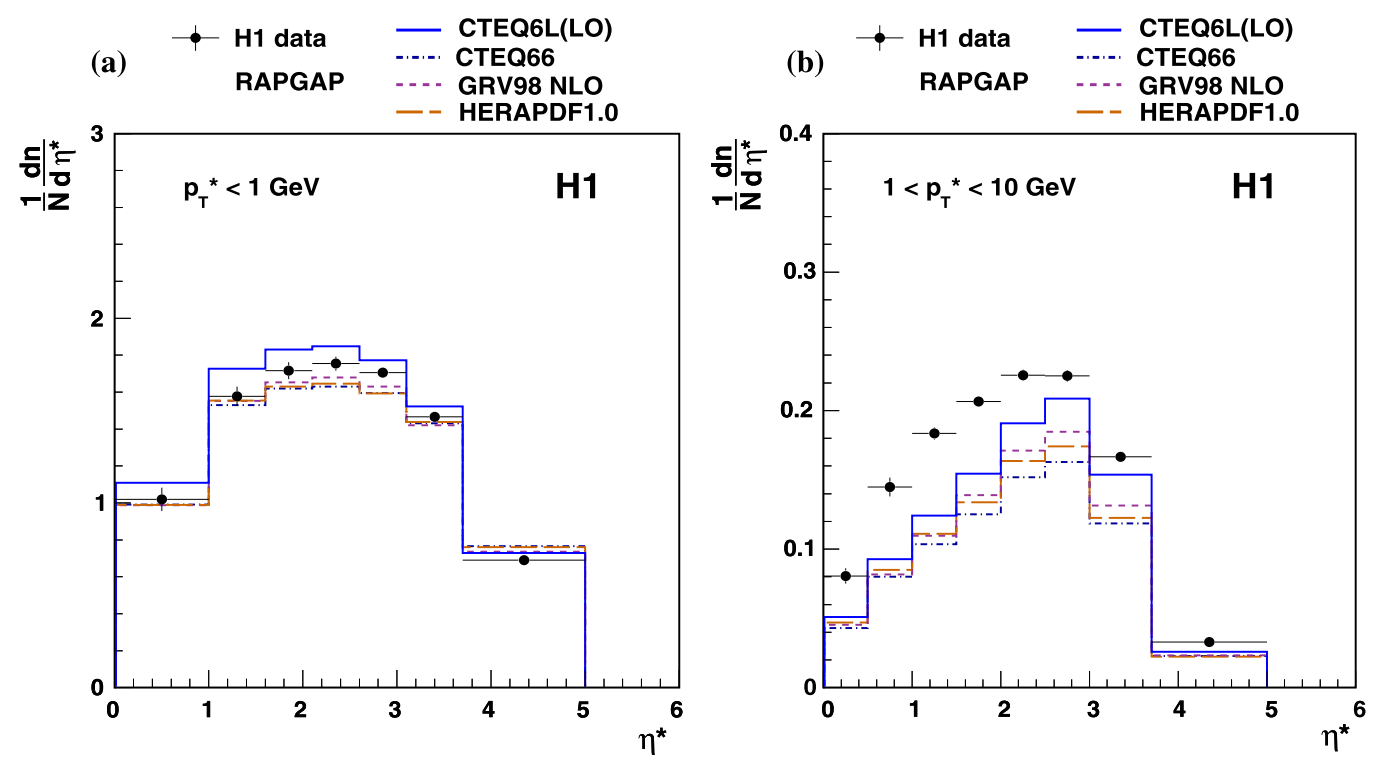

Fig. 3 Charged particle density as a function of $\eta^{*}$ for (a) $p_{T}^{*}<1 \mathrm{GeV}$ and for (b) $1<p_{T}^{*}<10 \mathrm{GeV}$ compared to RAPGAP predictions with different proton PDFs. The predictions are obtained using the ALEPH tune
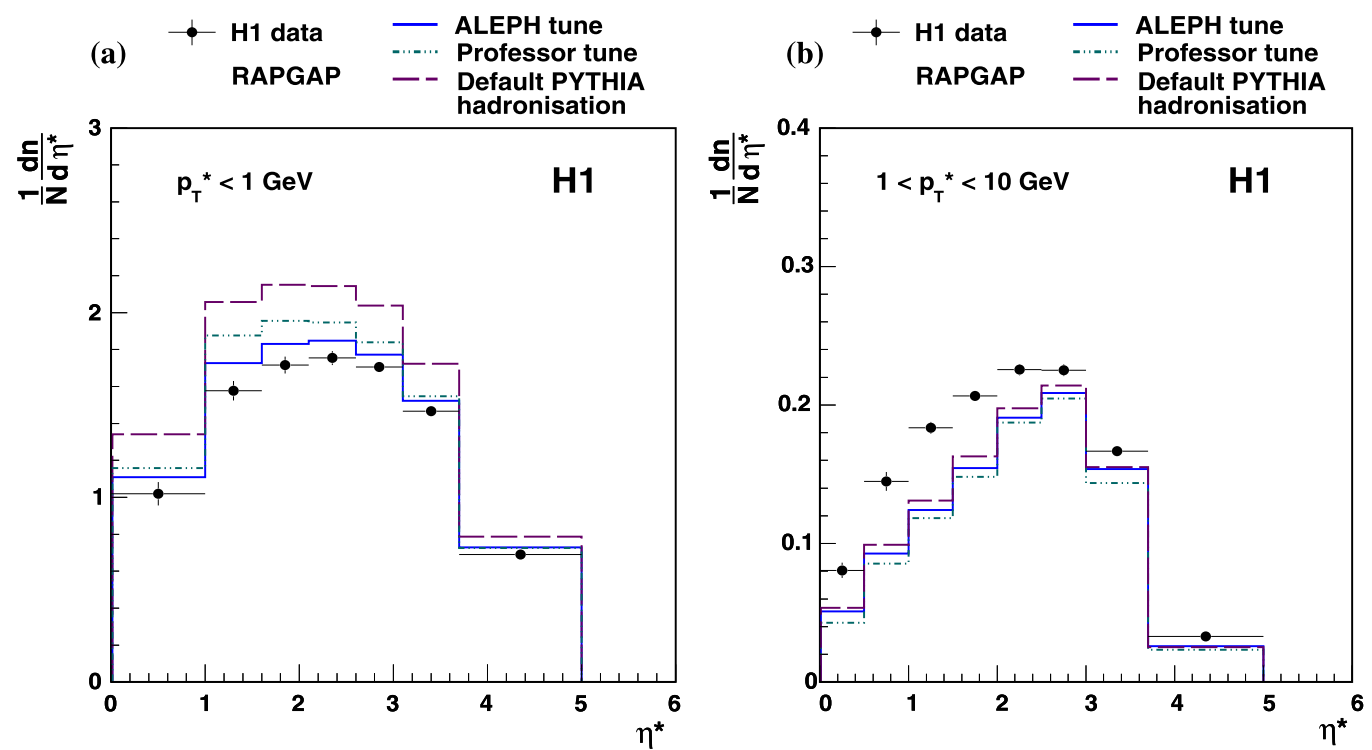

Fig. 4 Charged particle density as a function of $\eta^{*}$ for (a) $p_{T}^{*}<1 \mathrm{GeV}$ for (b) $1<p_{T}^{*}<10 \mathrm{GeV}$ compared to RAPGAP predictions for three different sets of fragmentation parameters. The predictions are obtained using CTEQ6L(LO) PDF

regions, degrading at high $p_{T}^{*}$ in the lowest $\left(x, Q^{2}\right)$ bin. Significant deviations of the RAPGAP predictions from data are observed in the central region at low $x$ and $Q^{2}$. The description becomes somewhat better at larger values of $x$ and $Q^{2}$. The same trend is observed for the current region, but the overall data description is better. Herwig++ fails to describe the measurements at high $p_{T}^{*}$ in the whole phase space. At lowest $x$ and $Q^{2}$ the spectrum is much softer than the one obtained with RAPGAP, while at high $x$ and $Q^{2}$ both predictions are similar. CASCADE describes the data in the lowest $x$ and $Q^{2}$ bin at high $p_{T}^{*}$ only.

\section{Conclusion}

This paper presents a study of charged particle production in ep collisions at low $Q^{2}$ measured with the $\mathrm{H} 1$ detector. The kinematic range of the analysis covers low photon virtualities, $5<Q^{2}<100 \mathrm{GeV}^{2}$, and small values of $x$, $10^{-4}<x<10^{-2}$. The analysis is performed in the hadronic centre-of-mass system. The charged particle densities as a function of pseudorapidity $\left(\eta^{*}\right)$ and transverse momentum $\left(p_{T}^{*}\right)$ are measured in bins of $x$ and $Q^{2}$. The charged particle densities as a function of pseudorapidity show different 

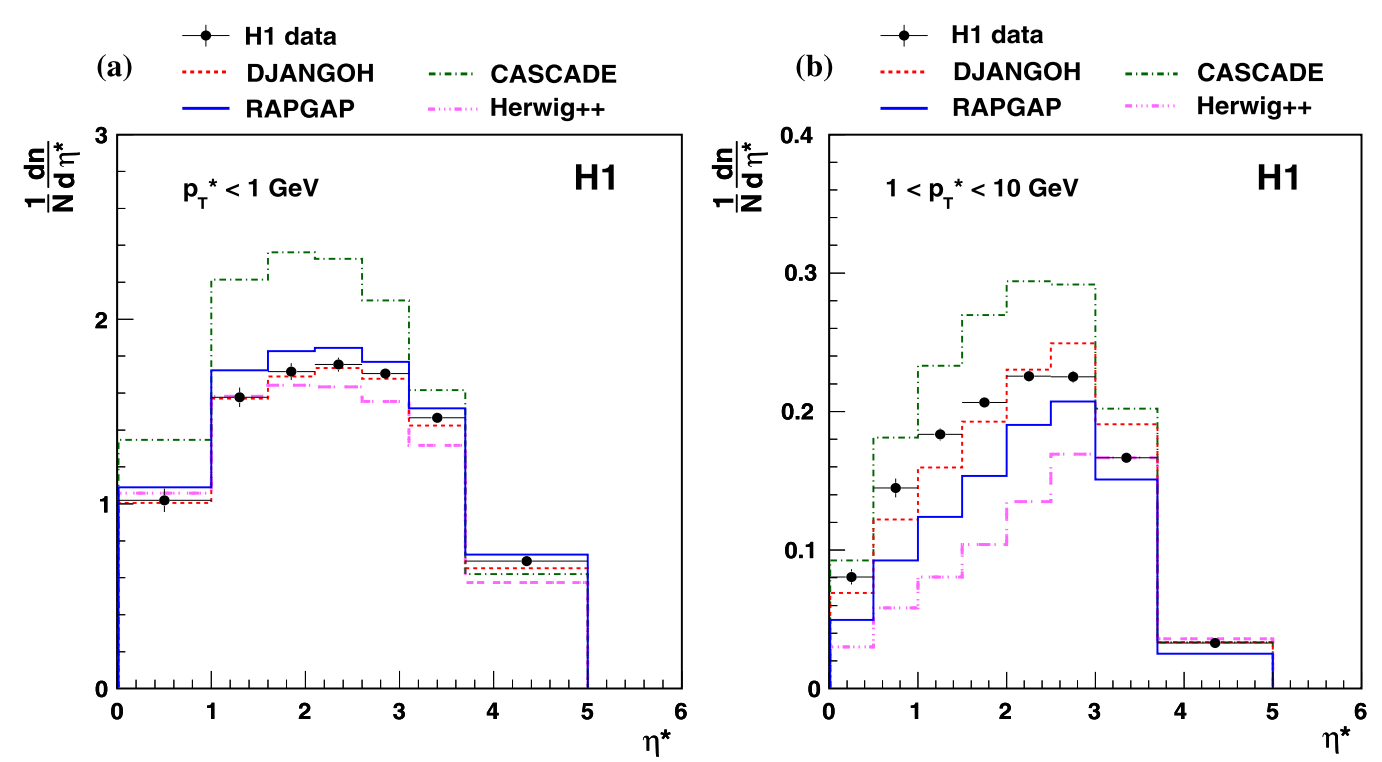

Fig. 5 Charged particle density as a function of $\eta^{*}$ for (a) $p_{T}^{*}<1 \mathrm{GeV}$ for (b) $1<p_{T}^{*}<10 \mathrm{GeV}$ compared to DJANGOH, RAPGAP, Herwig++ and CASCADE Monte Carlo predictions

Fig. 6 Charged particle density as a function of $\eta^{*}$ for $p_{T}^{*}<1 \mathrm{GeV}$ for eight intervals of $Q^{2}$ and $x$ compared to DJANGOH, RAPGAP, Herwig++ and CASCADE Monte Carlo predictions

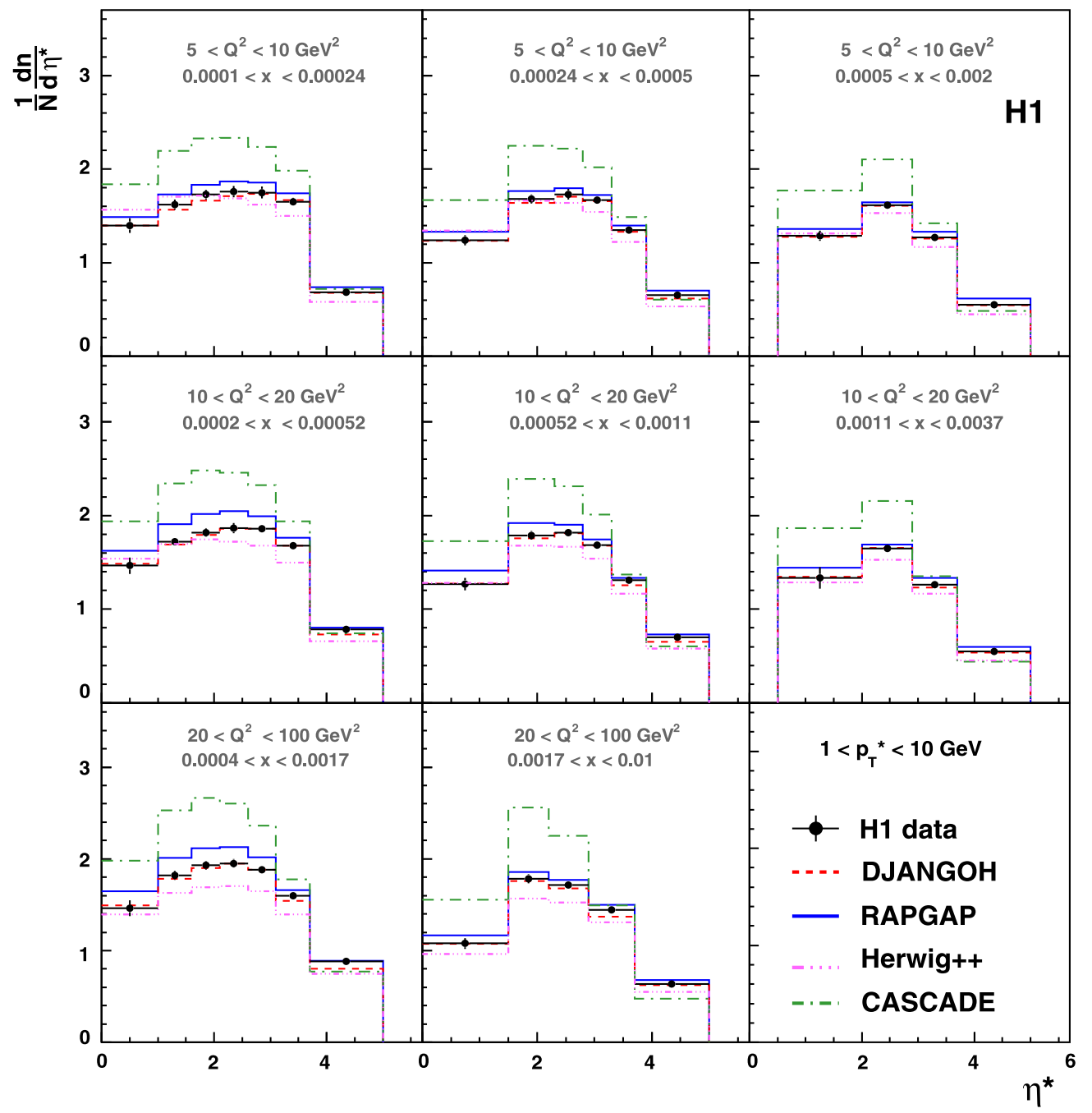


Fig. 7 Charged particle density as a function of $\eta^{*}$ for $1<p_{T}^{*}<10 \mathrm{GeV}$ for eight intervals of $Q^{2}$ and $x$ compared to DJANGOH, RAPGAP, Herwig++ and CASCADE Monte Carlo predictions
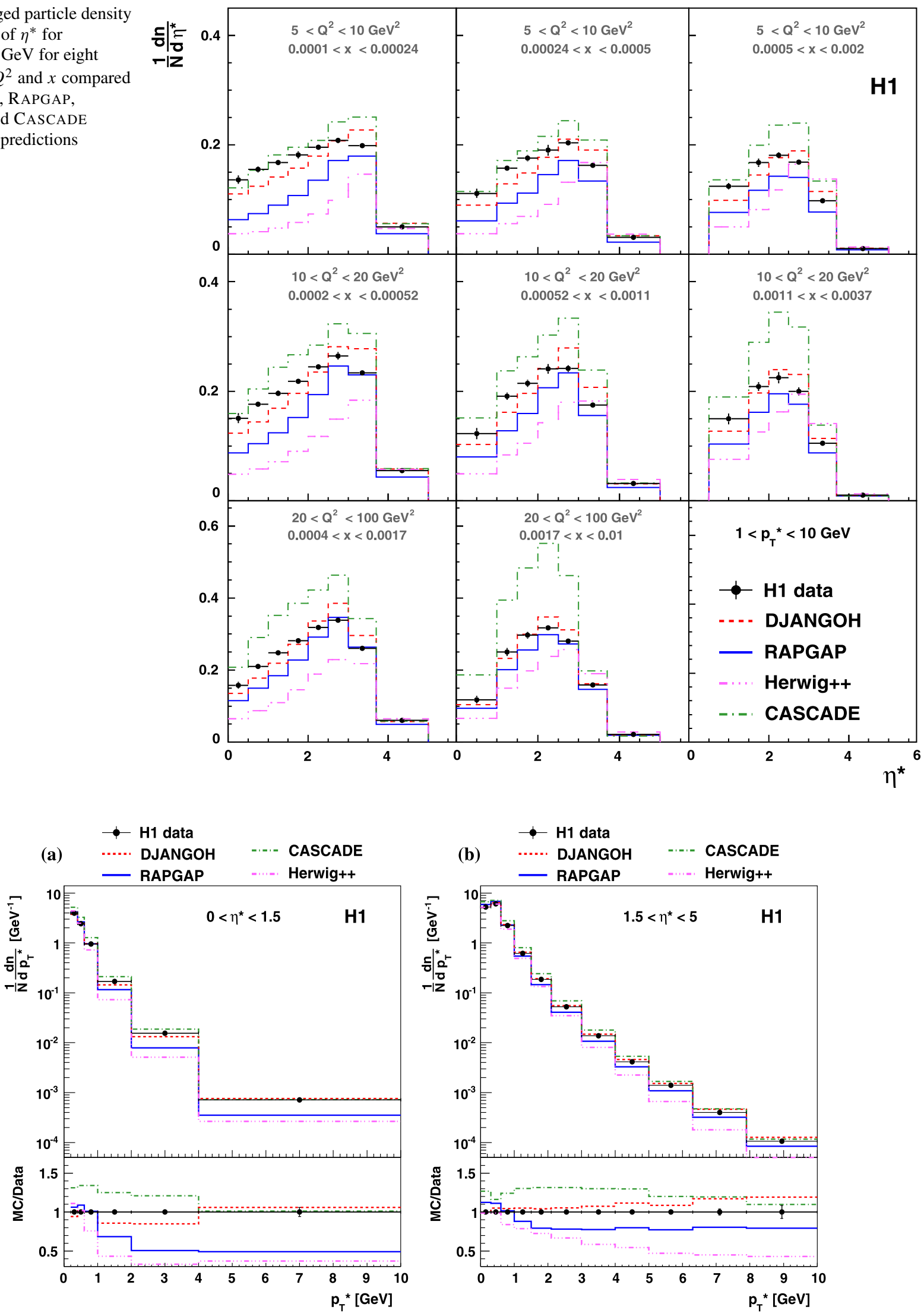

Fig. 8 Charged particle density as a function of $p_{T}^{*}$ in the ranges (a) $0<\eta^{*}<1.5$ and (b) $1.5<\eta^{*}<5$ compared to DJANGOH, RAPGAP, Herwig++ and CASCADE Monte Carlo predictions. The ratios of MC predictions to the measurements are shown on the bottom of the figure 
Fig. 9 Charged particle density as a function of $p_{T}^{*}$ in the range $0<\eta^{*}<1.5$ for eight intervals of $Q^{2}$ and $x$ compared to DJANGOH, RAPGAP, Herwig++ and CASCADE Monte Carlo predictions

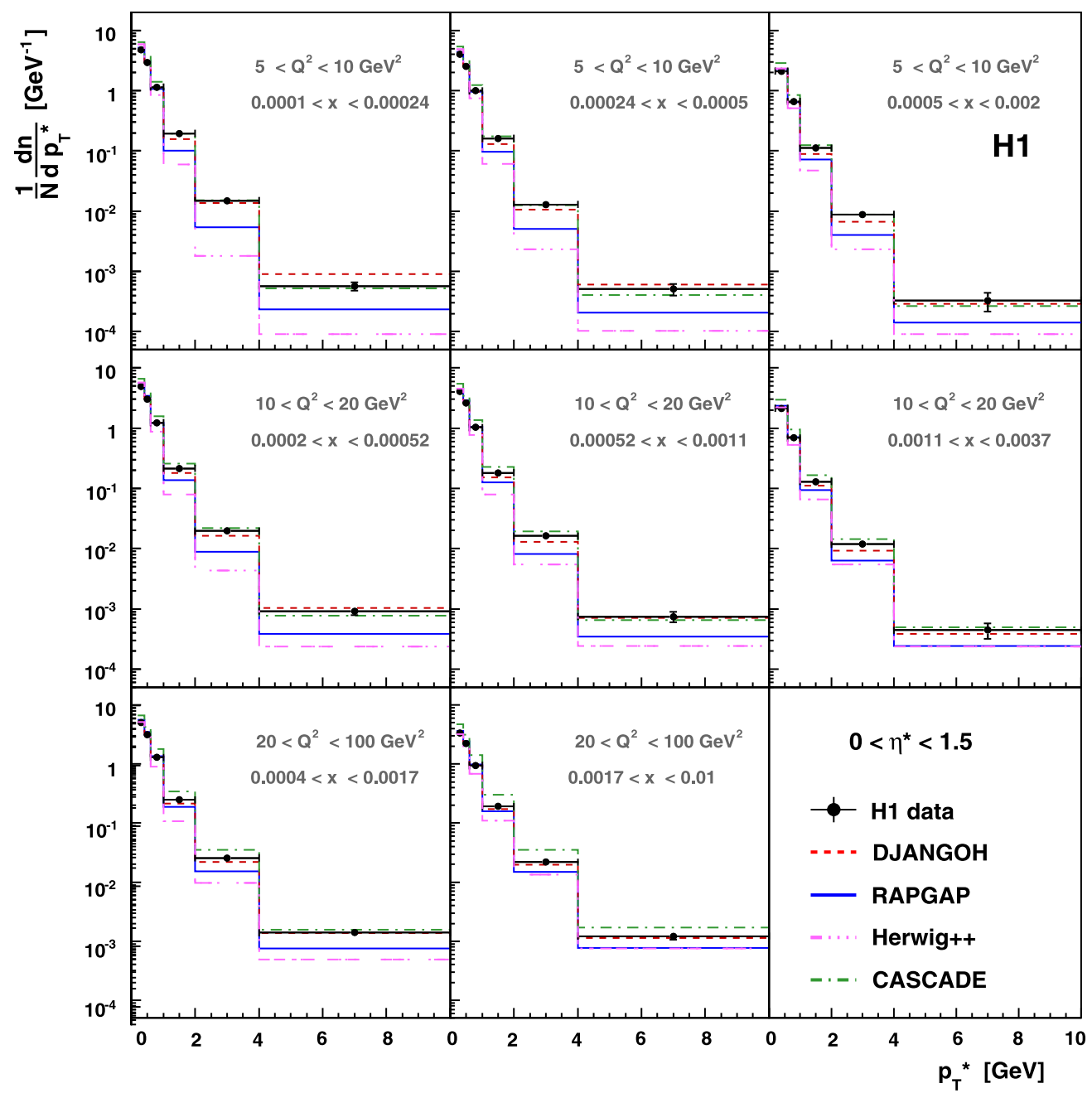

shapes, depending on the $p_{T}^{*}$ range. For $0<p_{T}^{*}<1 \mathrm{GeV}$ the density of particles is approximately constant for $1<$ $\eta^{*}<3.5$, while for $1<p_{T}^{*}<10 \mathrm{GeV}$ the density increases with increasing $\eta^{*}$ up to $\eta^{*} \approx 2.5$, a behaviour expected from the strong ordering of transverse momentum towards the hard scattering vertex. The charged particle densities as a function of transverse momentum show an $x$ dependence at small $\eta^{*}\left(0<\eta^{*}<1.5\right)$, such that the number of soft particles is decreasing with increasing $x$, while in the $1.5<\eta^{*}<5$ range this effect is less visible.

In order to relate the charged hadron spectra to the parton dynamics at small $x$, the data are compared to QCD models with different evolution approaches for simulating the parton cascade and with different hadronisation schemes. The data allow the validity of different models to be tested. At small $p_{T}^{*}$, the data are reasonably well described by DJAN$\mathrm{GOH}$ (based on the Colour Dipole Model), as well as by RAPGAP (based on the DGLAP shower evolution). At high $p_{T}^{*}$ and at low $\eta^{*}$, RAPGAP severely undershoots the data. The differences are most pronounced at lowest $x$ and $Q^{2}$, and decrease with increasing $x$ and $Q^{2}$ values. Herwig++ which is also based on DGLAP but uses a cluster fragmentation model reasonably describes the low $p_{T}^{*}$ region but undershoots the data even more than RAPGAP at high $p_{T}^{*}$. CASCADE (based on CCFM) gives a reasonable description only at the lowest $x$ and $Q^{2}$, but overall predicts higher charged particle densities than observed in data. The Colour Dipole Model implemented in DJANGOH is the best among the considered models and provides a reasonable description of the data.

Acknowledgements We are grateful to the HERA machine group whose outstanding efforts have made this experiment possible. We thank the engineers and technicians for their work in constructing and maintaining the $\mathrm{H} 1$ detector, our funding agencies for financial support, the DESY technical staff for continual assistance and the DESY directorate for support and for the hospitality which they extend to the non-DESY members of the collaboration. We would like to give credit to all partners contributing to the WLCG computing infrastructure for their support for the H1 Collaboration.

Open Access This article is distributed under the terms of the Creative Commons Attribution License which permits any use, distribution, and reproduction in any medium, provided the original author(s) and the source are credited. 
Fig. 10 Charged particle density as a function of $p_{T}^{*}$ in the range $1.5<\eta^{*}<5$ for eight intervals of $Q^{2}$ and $x$ compared to DJANGOH, RAPGAP, Herwig++ and CASCADE Monte Carlo predictions

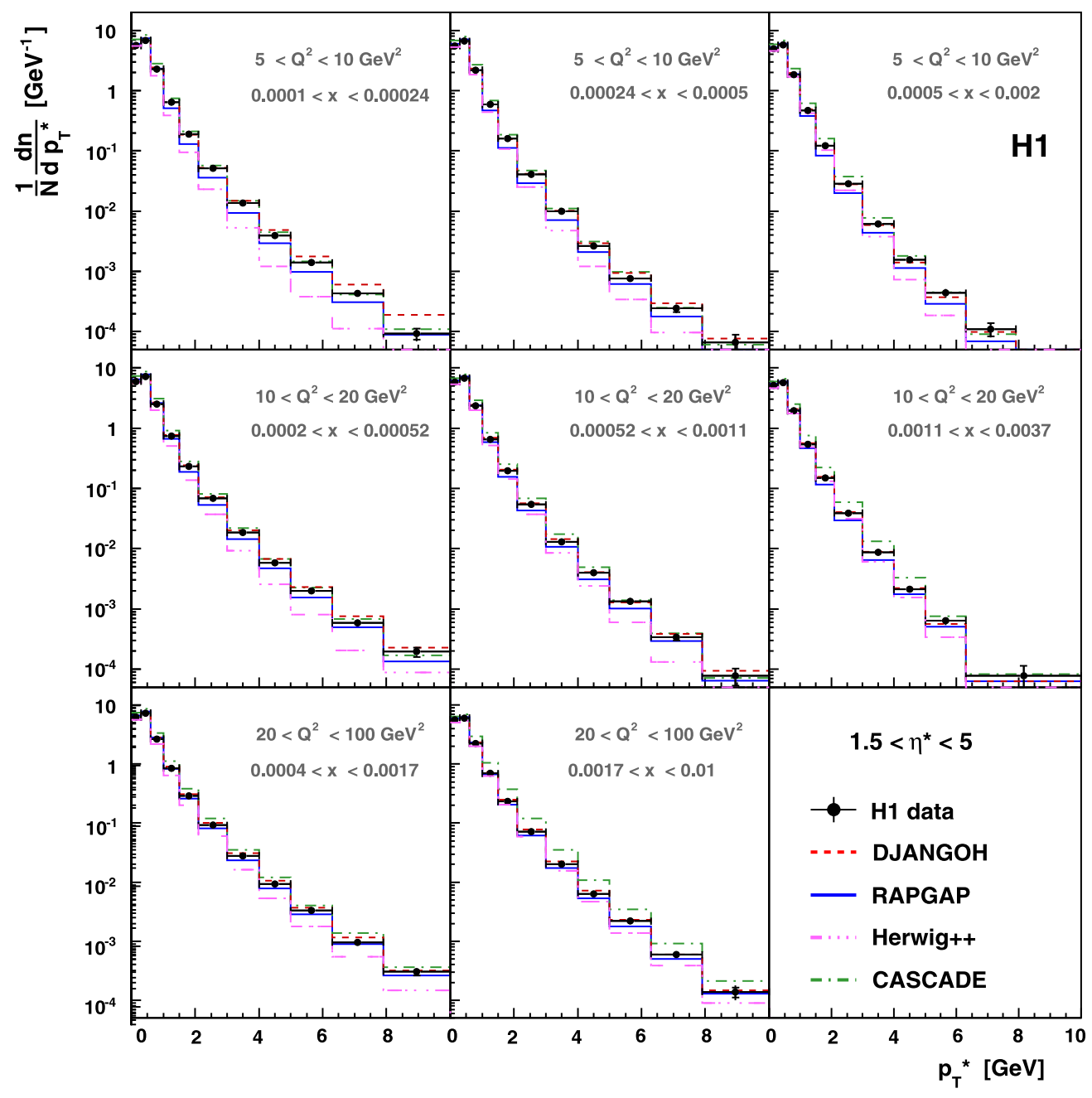

\section{References}

1. V. Gribov, L. Lipatov, Sov. J. Nucl. Phys. 15, 438 (1972)

2. V. Gribov, L. Lipatov, Sov. J. Nucl. Phys. 15, 675 (1972)

3. L. Lipatov, Sov. J. Nucl. Phys. 20, 94 (1975)

4. G. Altarelli, G. Parisi, Nucl. Phys. B 126, 298 (1977)

5. Y. Dokshitzer, Sov. Phys. JETP 46, 641 (1977)

6. E. Kuraev, L. Lipatov, V. Fadin, Sov. Phys. JETP 44, 443 (1976)

7. E. Kuraev, L. Lipatov, V. Fadin, Sov. Phys. JETP 45, 199 (1977)

8. Y. Balitsky, L. Lipatov, Sov. J. Nucl. Phys. 28, 822 (1978)

9. M. Ciafaloni, Nucl. Phys. B 296, 49 (1988)

10. M. Catani, F. Fiorani, G. Marchesini, Phys. Lett. B 234, 339 (1990)

11. S. Catani, F. Fiorani, G. Marchesini, Nucl. Phys. B 336, 18 (1990)

12. G. Marchesini, Nucl. Phys. B 445, 49 (1995)

13. F.D. Aaron et al. (H1 and ZEUS Collaborations), J. High Energy Phys. 1001, 109 (2010). arXiv:0911.0884

14. F.D. Aaron et al. (H1 Collaboration), J. High Energy Phys. 1209, 061 (2012). arXiv: 1206.7007

15. R.S. Thorne, Phys. Rev. D 73, 054019 (2006). hep-ph/0601245

16. A.D. Martin et al., Eur. Phys. J. C 63, 189 (2009), arXiv:0901. 0002

17. R.S. Thorne, arXiv: 1201.6180

18. H.L. Lai et al., Phys. Rev. D 82, 074024 (2010). arXiv:1007.2241

19. P. Nadolsky et al., Contribution to the Proceedings of the XX Workshop on Deep Inelastic Scattering and Related Subjects, Bonn, Germany, 26-30 March, 2012. arXiv:1206.3321
20. S. Forte et al., Nucl. Phys. B 834, 116 (2010). arXiv: 1001.2312

21. R.D. Ball et al. (NNPDF Collaboration), Nucl. Phys. B 849, 296 (2011). arXiv:1101.1300

22. R.D. Ball et al. (NNPDF Collaboration), Nucl. Phys. B 855, 153 (2012). arXiv:1107.2652

23. S. Alekhin, S. Moch, Phys. Lett. B 699, 345 (2011). arXiv:1011. 5790

24. S. Alekhin et al., Phys. Rev. D 81, 014032 (2010). arXiv:0908. 2766

25. S. Alekhin, S. Moch, arXiv:1107.0469

26. A. Aktas et al. (H1 Collaboration), Eur. Phys. J. C 33, 477 (2004). hep-ex/0310019

27. C. Adloff et al. (H1 Collaboration), Phys. Lett. B 415, 418 (1997). hep-ex/9709017

28. C. Adloff et al. (H1 Collaboration), Eur. Phys. J. C 12, 595 (2000). hep-ex/9907027

29. S. Aid et al. (H1 Collaboration), Eur. Phys. J. B 356, 118 (1995). hep-ex/9506012

30. A. Aktas et al. (H1 Collaboration), Eur. Phys. J. C 46, 27 (2006). hep-ex/0508055

31. S. Chekanov et al. (ZEUS Collaboration), Eur. Phys. J. C 52, 515 (2007). arXiv:0707.3093

32. F.D. Aaron et al. (H1 Collaboration), Eur. Phys. J. C 72, 1910 (2012). arXiv:1111.4227

33. A. Aktas et al. (H1 Collaboration), Eur. Phys. J. C 36, 441 (2004). hep-ex/0404009 
34. M. Kuhlen, Phys. Lett. B 382, 441 (1996). hep-ph/9606246

35. C. Adloff et al. (H1 Collaboration), Nucl. Phys. B 485, 3 (1997). hep-ex/9610006

36. H. Jung, RAPGAP 3.1. Comput. Phys. Commun. 86, 147 (1995)

37. K. Charchula, G.A. Schuler, H. Spiesberger, DJANGOH 1.4. Comput. Phys. Commun. 81, 381 (1994)

38. L. Lönnblad, ARIADne 4.10. Comput. Phys. Commun. 71, 15 (1992)

39. L. Lönnblad, Z. Phys. C 65, 285 (1995)

40. A.H. Mueller, Nucl. Phys. B 415, 373 (1994)

41. H. Jung, Comput. Phys. Commun. 143, 100 (2002)

42. H. Jung et al., CASCADE 2.2.0. Eur. Phys. J. C 70, 1237 (2010)

43. S. Gieseke et al., Herwig++2.5. arXiv: 1102.1672

44. L. D'Errico, P. Richardson, Eur. Phys. J. C 72, 2042 (2011). arXiv: 1106.2983

45. G. Marchesini, Nucl. Phys. B 445, 49 (1995). hep-ph/9412327

46. S. Catani, B.R. Webber, G. Marchesini, Nucl. Phys. B 349, 635 (1991)

47. S. Gieseke, P. Stephens, B. Webber, J. High Energy Phys. 0312, 045 (2003). hep-ph/0310083

48. R. Engel, Z. Phys. C 66, 203 (1995)

49. R. Engel, J. Ranft, Phojet 1.6. Phys. Rev. D 54, 4244 (1996). hep-ph/9509373

50. A. Capella et al., Phys. Rep. 236, 227 (1994)

51. J. Pumplin et al., J. High Energy Phys. 0207, 012 (2002). hep-ph/ 0201195

52. H. Jung, in Proceedings of the XII International Workshop on Deep Inelastic Scattering (DIS2004), ed. by D. Bruncko, J. Ferencei, P. Stríženec, Štrbské Pleso, Slovakia (2004), p. 299. hep-ph/ 0411287

53. A.D. Martin et al., Phys. Lett. B 531, 216 (2002). hep-ph/0201127

54. B. Andersson et al., Phys. Rep. 97, 31 (1983)

55. T. Sjöstrand et al., Comput. Phys. Commun. 82, 174 (1994). hep$\mathrm{ph} / 9508391$

56. T. Sjöstrand et al., Comput. Phys. Commun. 135, 238 (2001). hep$\mathrm{ph} / 0010017$

57. S. Schael et al. (ALEPH Collaboration), Phys. Lett. B 606, 265 (2005)

58. A. Buckley et al., Eur. Phys. J. C 65, 331 (2010). arXiv:0907.2973

59. B.R. Webber, Nucl. Phys. B 238, 492 (1984)

60. G. Marchesini, B.R. Webber, Nucl. Phys. B 310, 461 (1988)

61. A. Kwiatkowski, H. Spiesberger, H.J. Möhring, HeraCles 1.0. Comput. Phys. Commun. 69, 155 (1992)

62. R. Brun et al., GEANT 3. Technical Report CERN-DD/EE-84-1, 1987
63. A. Grebenyuk, PhD thesis, Hamburg University, 2012, available at http://www-h1.desy.de/publications/theses_list.html

64. I. Abt et al. (H1 Collaboration), Nucl. Instrum. Methods A 386, 310 (1997)

65. I. Abt et al. (H1 Collaboration), Nucl. Instrum. Methods A 386, 348 (1997)

66. R.D. Appuhn et al. (H1 SpaCal Group), Nucl. Instrum. Methods A 386, 397 (1997)

67. D. Pitzl et al., Nucl. Instrum. Methods A 454, 334 (2000). hep-ex/ 0002044

68. B. List, Nucl. Instrum. Methods A 501, 49 (2001)

69. J. Becker et al., Nucl. Instrum. Methods A 586, 190 (2008)

70. P.J. Laycock et al., J. Instrum. 7, T8003 (2012). arXiv:1206.4068

71. J. Kretzschmar, PhD thesis, Humboldt University, Berlin, 2008, available at http://www-h1.desy.de/publications/theses_list.html

72. I. Glushkov, PhD thesis, Humboldt University, Berlin, 2007, available at http://www-h1.desy.de/publications/theses_list.html

73. T. Nicholls et al. (H1 SpaCal Group), Nucl. Instrum. Methods A 374, 149 (1996)

74. B. Andrieu et al. (H1 Calorimeter Group), Nucl. Instrum. Methods A 336, 460 (1993)

75. B. Andrieu et al. (H1 Calorimeter Group), Nucl. Instrum. Methods A 336, 499 (1993)

76. U. Bassler, G. Bernardi, Nucl. Instrum. Methods A 426, 583 (1999). hep-ex/9801017

77. M. Peez, PhD thesis, Lyon University, 2003, available at http:// www-h1.desy.de/publications/theses_list.html

78. B. Portheault, PhD thesis, Paris XI ORSAY University, 2005, available at http://www-h1.desy.de/publications/theses_list.html

79. S. Hellwig, Dipl. thesis, Hamburg University, 2004, available at http://www-h1.desy.de/publications/theses_list.html

80. F.D. Aaron et al. (H1 Collaboration), Eur. Phys. J. C 63, 625 (2009). arXiv:0904.0929

81. D. Salek, PhD thesis, Charles Univ. Prague, DESY-THESIS-2011013, available at http://www-h1.desy.de/publications/theses_list. html

82. M. Brinkmann, PhD thesis, Hamburg University, 2010, available at http://www-h1.desy.de/publications/theses_list.html

83. P.M. Nadolsky et al., Phys. Rev. D 78, 013004 (2008). arXiv:0802. 0007

84. M. Glück, E. Reya, A. Vogt, Eur. Phys. J. C 5, 461 (1998). hep-ph/ 9806404 